\title{
Microglial inclusions and neurofilament light chain release follow neuronal a-synuclein lesions in long-term brain slice cultures
}

Melanie Barth 1,2,3, Mehtap Bacioglu1,2,3, Niklas Schwarz ${ }^{4}$, Renata Novotny ${ }^{1,2}$, Janine Brandes ${ }^{2,4}$, Marc Welzer ${ }^{1,2,3}$, Sonia Mazzitelli, ${ }^{1,2}$, Lisa M. Häsler ${ }^{1,2}$, Manuel Schweighauser ${ }^{1,2}$, Thomas V. Wuttke ${ }^{4,5}$,

Deborah Kronenberg-Versteeg ${ }^{1,2}$, Karina Fog ${ }^{6}$, Malene Ambjørn ${ }^{6}$, Ania Alik ${ }^{7}$, Ronald Melki ${ }^{7}$, Philipp J. Kahle ${ }^{1,8}$,

Derya R. Shimshek ${ }^{9}$, Henner Koch ${ }^{4,10}$, Mathias Jucker ${ }^{1,2^{*}}$ and Gaye Tanriöver ${ }^{1,2^{*}}$ (D)

\begin{abstract}
Background: Proteopathic brain lesions are a hallmark of many age-related neurodegenerative diseases including synucleinopathies and develop at least a decade before the onset of clinical symptoms. Thus, understanding of the initiation and propagation of such lesions is key for developing therapeutics to delay or halt disease progression.

Methods: Alpha-synuclein (aS) inclusions were induced in long-term murine and human slice cultures by seeded aggregation. An aS seed-recognizing human antibody was tested for blocking seeding and/or spreading of the aS lesions. Release of neurofilament light chain (NfL) into the culture medium was assessed.

Results: To study initial stages of a-synucleinopathies, we induced aS inclusions in murine hippocampal slice cultures by seeded aggregation. Induction of aS inclusions in neurons was apparent as early as 1 week post-seeding, followed by the occurrence of microglial inclusions in vicinity of the neuronal lesions at 2-3 weeks. The amount of aS inclusions was dependent on the type of aS seed and on the culture's genetic background (wildtype vs A53T-aS genotype). Formation of aS inclusions could be monitored by neurofilament light chain protein release into the culture medium, a fluid biomarker of neurodegeneration commonly used in clinical settings. Local microinjection of aS seeds resulted in spreading of aS inclusions to neuronally connected hippocampal subregions, and seeding and spreading could be inhibited by an aS seed-recognizing human antibody. We then applied parameters of the murine cultures to surgical resection-derived adult human long-term neocortical slice cultures from 22 to 61-yearold donors. Similarly, in these human slice cultures, proof-of-principle induction of aS lesions was achieved at 1 week post-seeding in combination with viral A53T-aS expressions.
\end{abstract}

Conclusion: The successful translation of these brain cultures from mouse to human with the first reported induction of human aS lesions in a true adult human brain environment underlines the potential of this model to study proteopathic lesions in intact mouse and now even aged human brain environments.

Keywords: Alpha-synuclein, Microglia, Neurofilament light chain, Slice culture

\footnotetext{
*Correspondence: mathias.jucker@uni-tuebingen.de; gaye.tanrioever@uni-

tuebingen.de

'DZNE, German Center for Neurodegenerative Diseases, 72076 Tuebingen, Germany

${ }^{2}$ Department of Cellular Neurology, Hertie Institute for Clinical Brain Research, University of Tuebingen, 72076 Tuebingen, Germany

Full list of author information is available at the end of the article
}

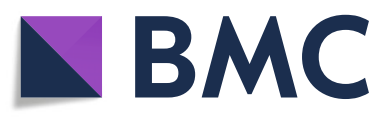

(c) The Author(s). 2021 Open Access This article is licensed under a Creative Commons Attribution 4.0 International License, which permits use, sharing, adaptation, distribution and reproduction in any medium or format, as long as you give appropriate credit to the original author(s) and the source, provide a link to the Creative Commons licence, and indicate if changes were made. The images or other third party material in this article are included in the article's Creative Commons licence, unless indicated otherwise in a credit line to the material. If material is not included in the article's Creative Commons licence and your intended use is not permitted by statutory regulation or exceeds the permitted use, you will need to obtain permission directly from the copyright holder. To view a copy of this licence, visit http://creativecommons.org/licenses/by/4.0/ The Creative Commons Public Domain Dedication waiver (http://creativecommons.org/publicdomain/zero/1.0/) applies to the data made available in this article, unless otherwise stated in a credit line to the data. 


\section{Background}

Synucleinopathy is a collective term for neurodegenerative diseases such as Parkinson's disease (PD), dementia with Lewy bodies (DLB), and multiple system atrophy (MSA). Initial evidence for a cell-non-autonomous mechanism underlying synucleinopathies came from the discovery of Lewy pathology in grafted cells in post mortem brains of PD patients [1,2]. Therefore, prion-like transmission of $\alpha \mathrm{S}$ lesions was proposed and the concept of seeded aggregation - a nucleation-dependent process similar to the one described for prions and amyloid-beta $(A \beta)$ - was suggested $[3,4]$. This concept gained further support by in vitro findings showing that the exogenous application of $\alpha \mathrm{S}$ fibrils induced Lewy body-like pathology in cultured neurons [5-8]. Similarly, induction and spreading of $\alpha \mathrm{S}$ lesions was reported in mice using recombinant $\alpha \mathrm{S}$ preformed fibrils ( $\alpha \mathrm{S}$ pff) or brain homogenates containing aggregated $\alpha \mathrm{S}$ [9-15].

Induction and propagation of proteopathic lesions are largely dependent on the interaction between the seed and the host environment [16]. Spreading of $\alpha \mathrm{S}$ lesions occurs along neural pathways and cell-to-cell transfer is cell-type dependent and promoted by neural activity [11, 17-19]. Thus, seed propagation is best studied in a living environment that closely mimics the adult or aged brain. While mice have been instrumental in the past to study such prion-like propagation of pathogenic seeds [16], mouse models are time-consuming and costly, and experimental manipulations to mechanistically understand propagation of seeds are challenging.

Studying propagation of pathogenic seeds in humans is even more intricate. Human stem cell-derived organoids and 3D-culture systems have been developed to mimic the human brain environment [20-22], however, they remain controversial regarding their purity, maturity, and cell subtype-identity. Grafting of human stem cell derived tissue into adult mice is another exciting new development $[23,24]$ but the influence of the murine host on the human transplant needs to be addressed further.

To study $\alpha$-synuclein $(\alpha S)$ inclusion formation, we induced seeding in long-term murine hippocampal slice cultures obtained from postnatal mouse brain, which preserves the complex cellular brain microenvironment. We found inclusions in both neurons and microglia with spreading of the lesions along neural pathways. Both induction and propagation could be blocked by a human $\alpha \mathrm{S}$ seed-recognizing antibody. To foster translation, progression of synucleinopathy and associated neurodegeneration in these cultures was monitored by assessing neurofilament light chain (NfL) protein levels in the culture medium, a biomarker used in preclinical animal studies as well as in clinical settings. We then applied the initial findings from the murine cultures to resection-derived adult human brain tissue cultures and succeeded in inducing $\alpha \mathrm{S}$ inclusions in a true adult human brain environment.

\section{Methods \\ Mice}

Wildtype C57BL/6 J, heterozygous Thy1-h[A53T] $\alpha \mathrm{S}$ transgenic (tg) [25], and $\mathrm{Snca}^{-/-}$(C57BL/6-Snca ${ }^{\text {tm1Rosl }}$ ) [26] pups were used for preparation of HSCs. Thy1$\mathrm{h}[\mathrm{A} 53 \mathrm{~T}] \alpha \mathrm{S}$ tg mice overexpress human mutant (A53T) $\alpha \mathrm{S}$ under control of a Thy1-promoter element. Experimental procedures were carried out in accordance with the veterinary office regulations of Baden-Wuerttemberg (Germany) and were approved by the local Animal Care and Use Committees.

Preparation of mouse hippocampal slice cultures (HSCs)

HSCs were prepared from pups at postnatal day 4-6 (P4-6) according to previously published protocols [27, 28]. After decapitation, brains of the pups were aseptically removed, the hippocampi were dissected and cut perpendicular to the longitudinal axis into $350 \mu \mathrm{m} \mathrm{sec}-$ tions with a tissue chopper. Carefully selected intact hippocampal sections were transferred into petri dishes containing an ice-cold buffer solution (minimum essential medium (MEM) supplemented with $2 \mathrm{mM}$ Gluta$\mathrm{MAX}^{\mathrm{m}}$ at $\mathrm{pH}$ 7.3). Three sections were placed onto a humidified porous polyethylene (PTFE) membrane insert (Merck Millipore, PICMORG50) and into a 6-well plate with $1.2 \mathrm{ml}$ culture medium (20\% heat-inactivated horse serum in $1 \mathrm{x}$ MEM complemented with GlutaMax ${ }^{\mathrm{Ts}}$ (1 $\mathrm{mM})$, ascorbic acid $(0.00125 \%)$, insulin $(1 \mu \mathrm{g} / \mathrm{ml}), \mathrm{CaCl}_{2}$ $(1 \mathrm{mM}), \mathrm{MgSO}_{4}(2 \mathrm{mM})$ and D-glucose $(13 \mathrm{mM})$ adjusted to $\mathrm{pH}$ 7.3) per well. HSCs were kept at $37^{\circ} \mathrm{C}$ in humidified $\mathrm{CO}_{2}$-enriched atmosphere. The culture medium was completely changed three times per week.

\section{Preparation and treatment of human slice cultures}

Approval (\#338/2014BO2) of the ethics committee of the University of Tuebingen as well as written informed consent was obtained from all patients and allowed spare tissue from resective brain surgery to be used for human slice cultures. Human neocortical slice cultures were prepared according to a previously published protocol [29]. Access tissue from temporal lobe was obtained from patients who had undergone resective brain surgery (patient 1: 61 years old, female, epilepsy due to ganglioglioma; patient 2: 49 years old, male, epilepsy due to cortical tubers; patient 3: 22 years old, male, epilepsy due to hippocampus sclerosis). Tissue was transported from the surgery room to the laboratory in oxygenated (95\% $\mathrm{O}_{2}$ and 5\% $\mathrm{CO}_{2}$ ) ice-cold artificial cerebrospinal fluid (aCSF; $110 \mathrm{mM} \mathrm{C}_{5} \mathrm{H}_{14} \mathrm{ClNO}, 26 \mathrm{mM} \mathrm{NaHCO} 3,10$ $\mathrm{mM}$ D-glucose, $11.6 \mathrm{mM}$ Na-ascorbate, $7 \mathrm{mM} \mathrm{MgCl}_{2}$, 
$3.1 \mathrm{mM}$ Na-pyruvate, $2.5 \mathrm{mM} \mathrm{KCl}, 1.25 \mathrm{mM} \mathrm{NaH}_{2} \mathrm{PO}_{4}$, and $0.5 \mathrm{CaCl}_{2}$ ). The tissue was cut into $250 \mu \mathrm{m}$ slices perpendicular to the cortical surface using a vibratome. Afterwards, the slices were kept in aCSF equilibrated with carbogen for $30 \mathrm{~min}$ at room temperature (RT) before they were transferred onto culture membrane inserts (Merck Millipore, PICMORG50) in a 6-well plate for cultivation. For the first hour following the slicing procedure, the slices were cultured in $1.5 \mathrm{ml}$ NSC media (48\% DMEM/F-12 (Life Technologies), 48\% Neurobasal (Life Technologies), 1x N-2 (Capricorn Scientific), 1x B27 (Capricorn Scientific), 1x Glutamax (Life Technologies), 1x NEAA (Life Technologies) + $20 \mathrm{mM}$ HEPES. From then on, human slice cultures were grown in human cerebrospinal fluid (hCSF) obtained from normal pressure hydrocephalus patients via lumbar puncture [29]. Approval of the ethics committee of the University of Tuebingen as well as written informed consent from all patients was obtained (\#338/2016A). The hCSF was collected, pooled and centrifuged at $2,000 \times \mathrm{g}$ at $4{ }^{\circ} \mathrm{C}$ for $10 \mathrm{~min}$. The supernatant was kept at $-80^{\circ} \mathrm{C}$ and thawed at RT before changing the medium. Cultures were kept in $1.5 \mathrm{ml} \mathrm{hCSF}$ at $37^{\circ} \mathrm{C}$ in humidified $\mathrm{CO}_{2}$-enriched atmosphere. The hCSF was completely changed three times per week. For $\alpha \mathrm{S}$ overexpression, AAV1/2-CMV/ CBA-human-A53T- $\alpha$-synuclein-WPRE-BGH-polyA

virus (AAV-hA53T- $\alpha$ S) (Vigene Biosciences, titer of $5 \times$ $10^{12}$ ) was injected evenly spaced, once per $5 \mathrm{~mm}^{2}$ of the slice, using a picospritzer (PDES-O2DX/NPI electronics, Tamm, Germany).

\section{Brain-derived aS seeds and aS pre-formed fibrils}

For brain-derived seeds, brain homogenates were prepared as previously described [13]. In short, fresh frozen pooled brainstems from spontaneously ill male and female Thy1-h[A53T] $\alpha \mathrm{S}$ tg mice (7-8 months old, $n=3$ brainstems per homogenate) and corresponding non-tg controls (20 months old, $n=2$ ) were used. Homogenisation at $10 \%(\mathrm{w} / \mathrm{v})$ was done in sterile PBS (Pre-cellys, $4 \times 10 \mathrm{~s}$ at $5500 \mathrm{rpm}$ ), followed by vortexing and centrifugation at $3,000 \mathrm{x}$ g for $5 \mathrm{~min}$ at $4{ }^{\circ} \mathrm{C}$. The supernatants were collected, aliquoted and stored at $-80^{\circ} \mathrm{C}$ until use.

Expression in E. coli, purification and quality control of human recombinant monomeric wt $\alpha \mathrm{S}$ was done as previously described [30]. For fibril formation, soluble wt $\alpha S$ was incubated in Tris- $\mathrm{HCl}$ buffer $(50 \mathrm{mM}$ Tris$\mathrm{HCl}, \mathrm{pH} 7.5,150 \mathrm{mM} \mathrm{KCl}$ ) at $37^{\circ} \mathrm{C}$ under continuous shaking for 5 days and formation of fibrils was assessed with Thioflavin $\mathrm{T}$. The fibrils were quality checked by transmission electron microscopy after negative staining before and after fragmentation as reported previously [30, 31]. In addition, the fibrils limited proteolytic pattern was also assessed as previously reported [30, 31].
Fragmentation was achieved by sonication using a sonotrode (sonication for $20 \mathrm{~min}, 0.5 \mathrm{~s}$ pulses; Sonicator UIS250V, equipped with VialTweeter, Hielscher Ultrasound Technology, Germany). The fibrils were imaged using a Jeol 1400 transmission electron microscope following their adsorption onto carbon-coated 200 mesh grids and negative staining with $1 \%$ uranyl acetate. The images were recorded using a Gatan Orius CCD camera (Gatan Inc., Pleasanton, CA, USA) (Supplementary Fig. 1). The average size of the fibrils after fragmentation $(47 \pm 5 \mathrm{~nm})$ was derived from length distribution measurements and their average molecular weight $(16,200$ $\mathrm{kDa}$ ) was derived from analytical ultracentrifugation sedimentation velocity measurements. The fibrils $(350 \mu \mathrm{M})$ were aliquoted ( $6 \mu \mathrm{l}$ per tube), flash frozen in liquid nitrogen and stored at $-80^{\circ} \mathrm{C}$. Fibrils were labeled with NHS-ester ATTO-550 as previously described [32].

\section{Seeding of the cultures}

Murine HSCs were kept for 10 days in vitro (DIV-10) without any experimental treatment. At DIV-10, $1 \mu \mathrm{l}$ of $\alpha \mathrm{S}$ pff (350 $\mu \mathrm{M}$ or dilutions thereof) or brainstem homogenate of Thy1-h[A53T] $\alpha \mathrm{S}$ tg or wt mice was pipetted on top of each culture. Human slice cultures were kept until DIV-3 without any experimental treatment. At DIV-3, $1 \mu \mathrm{l}$ of $\alpha \mathrm{S}$ pff $(35 \mu \mathrm{M})$ was pipetted on top of the culture.

Local injection of $\alpha \mathrm{S}$ pff into HSCs was performed into cornu ammonis 3 (CA3) at DIV-10. CA3 was identified as the region next to dentate gyrus (DG), which can be observed by light microscopy (dense cell layer resembling a horseshoe). Microinjection pipette (Science Products GmbH, GB150TF-10) was pulled using a micropipette puller (Sutter instruments, Model P-97; settings: 1 cycle, heat $=520$, pull $=50$, velocity $=50$, time $=250)$. To immediately visualise the injection, $0.3 \mu \mathrm{l}$ FastGreen dye (Carl Roth) was added to a $6 \mu \mathrm{l}$ aliquot of ATTO-550labelled or unlabelled $\alpha \mathrm{S}$ pff $(350 \mu \mathrm{M})$, upon loading the pipette. The loaded pipette was then inserted into the holder of a picospritzer (PDES-O2DX/ NPI electronics, Tamm, Germany), and the very end of the tip was carefully broken under visual guidance with sterile forceps, until a pressure pulse of $10 \mathrm{~ms}$ was able to release a small droplet of $\alpha \mathrm{S}$ pff from the tip. Post-injection microscopic examination of the broken tip revealed an opening size of 25$40 \mu \mathrm{m}$. HSCs in their 6-well-plates were placed under the light microscope, and the pipette was carefully inserted into CA3. With a pressure pulse of 10 to $20 \mathrm{~ms}$ ( $10 \mathrm{~ms}$ for larger tip openings, $20 \mathrm{~ms}$ for smaller tip openings), a small volume of $\alpha \mathrm{S}$ pff was injected. After having injected all slices, (approximately $1 \mathrm{~h}$ later) the medium was changed. 


\section{Antibody treatment}

The antibody HLu-3 is a human IgG1-recognizing $\alpha S$. The epitope of the antibody was determined to be amino acid 113-115 of human $\alpha$ S, using arrays of overlapping linear peptides at Pepscan (Pepscan Zuidersluisweg 28,243 RC Lelystad, The Netherlands). The affinity to human, mouse and cynomolgus $\alpha \mathrm{S}$ monomers is determined to be $31 \mathrm{nM}$ by surface plasmon resonance (BIAcore 3000 ). HLu-3 has approximately a 200 -fold avidity shift in the binding to fibrillated forms of human $\alpha \mathrm{S}$ (determined by competition ELISA). HLu-3 has no crossreactivity to beta- \& gamma-synuclein. Reference item used in the study was a negative isotype-matched control, anti-HIV-1 gp120 human IgG1 antibody (b12).

HLu-3 or control antibody were either mixed with human $\alpha$ S pff (1:1 in PBS to obtain a final concentration of $35 \mu \mathrm{M})$ and drop seeded on top of each HSC at DIV-10, or added to the medium. For the latter, antibodies were added to pre-warmed culture medium upon medium change (final concentration of $350 \mathrm{nM}$ ). Depending on experimental scheme, antibody-supplemented medium was used 7 days prior to the application of $\alpha \mathrm{S}$ pff seeds (-7 days post-injection, dpi) or $1 \mathrm{~h}$ after $\alpha \mathrm{S}$ pff seeds $(0$ dpi). Thereafter, the cultures were treated with antibody-supplemented medium with every medium change.

\section{Histological analysis of cultures}

Cultures were fixed with $4 \%$ paraformaldehyde (PFA) in $\mathrm{PBS}$ at $\mathrm{pH} 7.4$ for $2 \mathrm{~h}$ (HSCs) or for $24 \mathrm{~h}$ (human slice cultures). After fixation, HSCs were rinsed 3 times with $0.1 \mathrm{M}$ PBS for $10 \mathrm{~min}$ and stored in PBS at $4{ }^{\circ} \mathrm{C}$ for up to 1 month until sectioning. Human slice cultures were rinsed 3 times with PBS supplemented with 0.2\% TritonX-100 (PBST) and incubated with PBST overnight for permeabilization. The membrane carrying the fixed HSCs was cut out and mounted onto a planar agar block. With a vibratome (Leica VT 1000S Vibratome, Leica Bio-systems), the cultures were sliced into $50 \mu \mathrm{m}$ sections. Typically, 5-6 intact sections per HSC were obtained and collected in PBS to be stained within 1 week. Human slice cultures were stained unprocessed.

Antigen retrieval was performed by heating the sections in $10 \mathrm{mM}$ citrate buffer $(1.8 \mathrm{mM}$ citric acid, 8.2 $\mathrm{mM}$ trisodium citrate, $\mathrm{pH} 6.0$ ) at $90^{\circ} \mathrm{C}$ for $35 \mathrm{~min}$ (HSCs) or overnight at $4{ }^{\circ} \mathrm{C}$ and subsequently $30 \mathrm{~s}$ at $90^{\circ} \mathrm{C}$ (human slice cultures). Sections were blocked with $5 \%$ NGS (HSCs, $2 \mathrm{~h}$ ) or 1\% NGS (human slice cultures, overnight at $4{ }^{\circ} \mathrm{C}$ ) and $0.3 \%$ PBST. For detection of $\alpha \mathrm{S}$ phosphorylated at Ser-129, a rabbit monoclonal pS129 antibody (Abcam, EP1536Y, Cat\# ab51253, 1:1,000) was used. For microglia detection rabbit monoclonal iba1 (Wako Chemicals GmbH, Cat\# 019-19,741, 1:250) and for neuronal staining and structure mouse monoclonal
NeuN antibody (Millipore GmbH, Cat\# MAB377, 1:500) and chicken anti-MAP2 (Abcam, Cat\# ab5392, 1:500) were used. Following Alexa-fluorophore-conjugated secondary antibodies were applied in a concentration of 1 : 250: goat-anti-rabbit Alexa-568 (Thermo Fisher, Cat\# A11011); goat-anti-mouse Alexa-488 (Thermo Fisher, Cat\# A11001); goat-anti-mouse Alexa-568 (Invitrogen, Cat\# A11004); goat-anti-rabbit Alexa-633 (Thermo Fisher, Cat\# A21070); goat-anti-chicken Alexa-488 (Invitrogen, Cat\# A21467). DAPI counterstaining was performed at a concentration of 1:500.

For staining with amyloid binding dyes pentamer formyl thiophene acetic acid (pFTAA) [33, 34] and thioflavin $\mathrm{S}$ (ThioS), sections were incubated for $1 \mathrm{~h}$ with either freshly prepared pFTAA $(1.5 \mathrm{mM}$ in de-ionized water, used at 1:500 in PBS) or ThioS (Sigma-Aldrich, Cat\# T1892; $1 \%$ w/v ThioS in milliQ $\mathrm{H}_{2} \mathrm{O}$ ). ThioSstained sections were washed $2 \mathrm{x}$ in $70 \% \mathrm{EtOH}$ and for $10 \mathrm{~min}$. Slices were transferred on glass slides and coverslipped with Dako Fluorescence mounting medium (Biozol Diagnostika, Cat\# S3023).

Sections were analyzed using an Axioplan2 imaging microscope (Zeiss, Jena, Germany) and digitised with an AxioCam HRm black and white camera (Zeiss) using AxioVision 4.8 software (Zeiss). With a Plan Neofluar $10 x / 0.50$ objective lens (Zeiss), 16-bit RGB mosaics of the whole culture were obtained with a resolution of 170 pixels $/ \mu \mathrm{m}$. High resolution images were acquired using a Zeiss LSM 510 META (Axiovert $200 \mathrm{M}$ ) confocal microscope with an oil immersion $40 \times / 1.3$ or $63 \times / 1.4$ Plan Apochromat objective and LSM software 4.2 (Carl Zeiss). Sequential excitation of fluorophores was performed using lasers with the wavelength $405 \mathrm{~nm}$ (DAPI), $488 \mathrm{~nm}$ (Alexa-488 coupled secondary antibodies, ThioS, pFTAA), $543 \mathrm{~nm}$ (Alexa-568 coupled secondary antibodies, pFTAA), and $633 \mathrm{~nm}$ (Alexa-633 coupled antibodies).

\section{Quantification of immunohistochemical stainings}

For quantification of pS129- and ThioS-positive inclusions in HSCs, whole culture mosaic images were acquired on an Axioplan2 imaging microscope as described above. Cultures with sectioning artefacts or cultures that were injected in the wrong site (e.g. into CA1 instead of CA3) were excluded from the analysis with FIJI Image (version 2.1.0/1.53c). Images were blinded, colour channels were split, background was subtracted (rolling ball radius 50 pixels), and the intensity threshold was manually adjusted. To exclude unspecific staining of ThioS, the particle size of signal in the green channel was limited to $20-200 \mu \mathrm{m}^{2}$. On each mosaic, the percentage of pS129- and ThioS-positive signal over the whole culture was calculated. 
To quantify percentage of pS129 and ThioS-signal in the injection site and other hippocampal subregions, images were blinded, regions of interest (ROIs) for CA3, CA2, CA1, DG and subiculum were selected based on the brain map of the Allan Mouse Brain Atlas (2004) (P56, coronal) and stored in FIJI before channels were split. Background was subtracted and intensity threshold for pS129 or ThioS was adjusted for the whole culture. For each ROI, the percentage of pS129- and ThioSpositive area over the respective ROI area was calculated.

\section{Heatmaps}

To demonstrate spreading through hippocampal subregions, heatmaps were prepared by illustrating the mean percentage of $\mathrm{pS} 129$ - or ThioS area obtained in the ROI analysis (see "Quantification of immunohistochemical stainings") by a colour code. Using Microsoft Excel (v.16), mean values were assigned to a colour based on a 3-colour-scale from white (\#FFFFFF) via yellow (\#FDBF2D) to red (\#BE0712). The colours were identified using Adobe Photoshop CS5 (v.12) and applied to the respective area of a hippocampal map in Adobe Illustrator CS5 (v.15).

\section{Biochemical analysis of cultures}

Slice culture homogenates were prepared from treated or untreated cultures. Cultures were removed from the membrane, pooled $(n=16)$ and immediately frozen on dry ice and stored at $-80^{\circ} \mathrm{C}$ until use. Frozen slice cultures were homogenised with a syringe in $160 \mu$ l sterile PBS, aliquoted and stored at $-80^{\circ} \mathrm{C}$ until further use. For immunoassays (Western blotting) homogenates in PBS were shifted to high salt (HS) buffer $(50 \mathrm{mM}$ Tris$\mathrm{HCl} \mathrm{pH} \mathrm{7.5,} 750 \mathrm{mM} \mathrm{NaCl}, 5 \mathrm{mM}$ EDTA, $1 \%$ phosphatase and protease inhibitor cocktails). $100 \mu \mathrm{L}$ of homogenate were incubated on ice in $N$-lauroylsarcosyl (Sigma, Cat\# 61747, Saint-Quentin-Fallavier, France) at a final concentration of $10 \%$, and were left on ice for 15 min before they were loaded on a $10 \%$ sucrose cushion and ultracentrifuged at $186,000 \times \mathrm{g}$ for $1 \mathrm{~h} 10 \mathrm{~min}$ at $4{ }^{\circ} \mathrm{C}$. The supernatant was collected, and the pellet was resuspended in sample buffer, and sample buffer was also added to the supernatant. Proteins were separated in a $4-12 \%$ SDS NuPage Gel and electroblotted onto Amersham nitrocellulose membranes (VWR International Merck Eurolab, Cat\# 10600001). Membranes were incubated with $0.4 \%$ PFA for $30 \mathrm{~min}$, and then saturated with $5 \%$ dry milk in $0.1 \%$ PBS-Tween20 (0.1\%). Monoclonal rabbit antibody against pS129 (AbCam, Cat\# ab51253) at 1:1,000 dilution to detect phosphorylated $\alpha \mathrm{S}$ species, monoclonal mouse antibody against $\alpha \mathrm{S}$ (BD Transduction Laboratories, Cat\# 610786) at 1:1,000 dilution to detect total $\alpha \mathrm{S}$, and rabbit $\beta$-actin antibody
(AbCam, Cat\# ab8227) as a loading control were used. Membranes were then incubated for $1 \mathrm{~h}$ with anti-rabbit antibody or anti-mouse antibody at 1:20,000 for $1 \mathrm{~h}$ at RT. Samples were visualized with chemiluminescence using SuperSignal West Dura Extended or Pico (both Thermo Scientific).

\section{Immunoassay for total aS measurements in brain homogenate}

For $\alpha \mathrm{S}$ measurements, brain homogenates were extracted as follows: aliquots were thawed on ice, mixed 1: 3.2 with cold formic acid (FA) (minimum 96\% purity; Sigma, St. Louis, MO, USA), sonicated for $35 \mathrm{~s}$ at $4{ }^{\circ} \mathrm{C}$, and spun at 25,000 g at $4 \mathrm{C}$ for $1 \mathrm{~h}$. The supernatant was equilibrated (1:20) in neutralization buffer ( $1 \mathrm{M}$ Tris base, $0.5 \mathrm{M} \mathrm{Na}_{2} \mathrm{HPO}_{4}, 0.05 \% \mathrm{NaN}_{3}$ ).

Concentrations of human $\alpha \mathrm{S}$ in brain homogenates were determined with an electrochemiluminescencelinked immunoassay using the MSD Human $\alpha$-Synuclein Kit (Meso Scale Discovery, Gaithersburg, MD, USA), or by Single Molecule Array (Simoa) technology using the Simoa $^{\mathrm{mi}}$ Human Alpha-Synuclein Discovery Kit (Quanterix, Billerica, MA, USA) according to manufacturer's instructions. FA-soluble brain homogenates were diluted up to 1:10,000 in Diluent 35 (Meso Scale Discovery) or 1:100 in Alpha-Synuclein Sample Diluent (Simoa) before the measurement, and analyzed in duplicates on a Mesoscale Sector Imager 6000 or a Simoa HD-1 Analyzer. MSD DISCOVERY WORKBENCH software 3.0 or Simoa Software Version 1.5 for HD-1 Analyzer was used for data analysis. Internal reference samples were used as controls on every plate.

\section{Immunoassay for aggregated aS measurements in brain homogenate and pre-formed fibrils}

$\alpha \mathrm{S}$ aggregates were measured using a HTRF-FRET assay developed by Cisbio (\#6FASYPEG, Cisbio). $\alpha \mathrm{S}$ pff and tg brain homogenate were serially diluted and a HTRFFRET signal measured on a PHERAstar (BMG LAB$\mathrm{TECH}$ ) using $337 \mathrm{~nm}$ laser excitation, simultaneous dual emission $665 \mathrm{~nm} / 620 \mathrm{~nm}$ and HTRF technology. Data is reported as $665 \mathrm{~nm} / 620 \mathrm{~nm} \times 10,000 . \alpha \mathrm{S}$ pff needed to be diluted $>50,000$ fold to be on the proper side of the hook effect of the assay, whereas the tg brain homogenate did not show hook effect issues at any dilutions. For $\alpha \mathrm{S}$ pff, a dilution of 204,800-fold resulted in a signal of approximately 20,000, whereas the tg brain homogenate only was diluted 25 -fold to reach a similar aggregation level. The difference in dilutions (e.g. 204,800-fold vs 25 -fold) to reach a similar aggregation level was used to estimate the relative $\alpha \mathrm{S}$ aggregation level per volume of sample. Results over several dilutions were combined to reach the final result of $8,000 \times$ more aggregates in $\alpha \mathrm{S}$ pff relative to tg brain homogenate per volume. 


\section{NfL immunoassay}

Culture medium was collected, aliquoted, and kept at $-80^{\circ} \mathrm{C}$ until use. NfL concentrations were determined by Single Molecule Array (Simoa) technology using the highly sensitive Simoa ${ }^{\mathrm{Tm}}$ NF-Light Advantage Kit (Quanterix, Billerica, MA, USA) according to manufacturer's instructions [35]. Medium samples were pre-diluted 1:10 or 1:50 in NF-Light sample diluent and measured in duplicates on a Simoa HD-1 Analyzer (Quanterix). Internal reference samples were used as controls on every plate. Detection limit of NfL was $0.038 \mathrm{pg} / \mathrm{ml}$.

\section{Culture thickness}

While sectioning the freshly fixed cultures with a vibratome (see above), the amount of $50 \mu \mathrm{m}$ sections was assessed. Although the first and the last section sometimes were not exactly $50 \mu \mathrm{m}$, the amount of sections $\times$ $50 \mu \mathrm{m}$ equals roughly the culture thickness at the time of fixation.

\section{Intracerebral injection of aS tg mice}

Male and female 3-4-month-old Thy1-h[A53T] $\mathrm{SS}$ tg mice were anesthetized using a mixture of ketamine $(100 \mathrm{mg} / \mathrm{kg}$ body weight) and xylazine $(10 \mathrm{mg} / \mathrm{kg}$ body weight) in saline. $2.5 \mu \mathrm{l}$ of brain homogenate (see above) was then infused bilaterally into the dorsal hippocampus (AP $-2.5 \mathrm{~mm}, \mathrm{~L} \pm 2.0 \mathrm{~mm}$, DV $-1.8 \mathrm{~mm}$ ) by stereotactic injection. Injection speed was $1.25 \mu \mathrm{l} / \mathrm{min}$, and the needle was slowly removed after being kept in place for an additional $2 \mathrm{~min}$. The surgical area was cleaned with sterile saline and the incision was sutured. Mice were kept under infrared light for warmth and monitored until recovery from anaesthesia.

After incubation periods of up to 30 days, mice were perfused for $5 \mathrm{~min}$ with ice-cold PBS. Brains were removed and immersion-fixed in $4 \%$ PFA in $0.1 \mathrm{M} \mathrm{PB}$ at $\mathrm{pH} 7.4$ for $48 \mathrm{~h}$, and then placed in $30 \%$ sucrose in PBS for $48 \mathrm{~h}$. Brains were frozen in 2-methylbutane, cooled with dry ice, and then serially cut into $25 \mu \mathrm{m}$ sagittal sections using a freezing-sliding microtome. The sections were collected in cryoprotectant (35\% ethylene glycol, $25 \%$ glycerol in PBS) and stored at $-20^{\circ} \mathrm{C}$ until use. Immunohistochemical staining of $\alpha \mathrm{S}$ inclusions was done as described for HSCs (see above).

\section{Statistical analysis}

All statistics were performed using PRISM software (GraphPad v.9). Data were tested for normality using the Shapiro-Wilk test. If the groups passed the normality test and only two groups were compared, an unpaired two-tailed t-test was applied. If the two groups did not pass the normality test, the non-parametric MannWhitney test was used for comparison. To estimate the influence of two different variables, a two-way-ANOVA was performed. If ANOVA revealed significant effects, post hoc Bonferroni's multiple comparisons test was used. The mean and standard error of the mean (SEM) are reported for each experimental group.

\section{Results}

Induction of aS inclusions in murine hippocampal slice cultures

Murine hippocampal slice cultures (HSCs) were prepared from postnatal days 4-6 Thy1-h[A53T] $\alpha$ S tg or wt mice (tg HSCs vs wt HSCs) and grown in culture for 10 days to stabilize. $\alpha S$ inclusions were induced by onetime application of $\alpha \mathrm{S}$ seed-rich aged Thy1-h[A53T] $\alpha \mathrm{S}$ transgenic $(\mathrm{tg})$ mouse brain homogenate or alternatively by synthetic $\alpha \mathrm{S}$ preformed fibrils (pff) (Fig. 1A). Five weeks later, all cultures had developed abundant perikaryal and neuritic $\alpha \mathrm{S}$ lesions stained with an antibody specific for phosphorylation at serine 129 (pS129) (Fig. $1 \mathrm{~B}$ and $\mathrm{C}$ ), a surrogate marker of neuronal $\alpha \mathrm{S}$ assembly [37]. The neuronal $\alpha \mathrm{S}$ inclusions stained also positive for the amyloid-binding dye pFTAA, but much less so for Thioflavin S (ThioS). However, there were numerous ThioS-positive inclusions that were largely localized to microglia (Fig. 1C), consistent with recent observations in $\alpha \mathrm{S}$ tg mice [36]. The microglial inclusions were always in vicinity of the neuronal inclusions. They appeared wool-like and were localized mainly close to the nucleus with an overall appearance different from the neuronal inclusions (Fig. 1B and C). For both neuronal and microglial lesions, the induction was faster and more abundant in tg HSCs compared to wt HSCs (Fig. 1D). Untreated control cultures, cultures treated with wt brain homogenate, and $\mathrm{Snca}^{-/-}$cultures did not develop any $\alpha \mathrm{S}$ inclusions (Fig. 1D; Supplementary Fig. 2). Analysis of the sarkosyl-insoluble HSCs fractions of both $\alpha \mathrm{S}$ pff and tg brain homogenate-treated cultures confirmed the presence of aggregated $\alpha \mathrm{S}$ very similar to that in the brain homogenates (Fig. 1E).

\section{Induction of aS inclusions is dependent on seed concentration and incubation time}

Titration experiments revealed increased neuronal and microglial inclusions with increasing concentration of the seeds (Fig. 2A, B). Tg brain homogenate appeared approximately $100 \mathrm{x}$ less potent (Fig. 2B) but also contained 1,000 $\mathrm{x}$ fewer monomeric $\alpha \mathrm{S}$ equivalents (typically $5 \mu \mathrm{g} / \mathrm{ml}$; measured by immunoassays after formic acid treatment) compared to undiluted $\alpha \mathrm{S}$ pff $(350 \mu \mathrm{M}$ or 5 $\mathrm{mg} / \mathrm{ml}$ ). This observation suggests that tg brain-derived $\alpha \mathrm{S}$ seeds are more seeding potent than their synthetic counterparts, which is also in line with direct measurements of $\alpha \mathrm{S}$ aggregates (Supplementary Fig. 3).

Neuronal $\alpha \mathrm{S}$ lesions appeared in tg HSCs after 1 week and decreased slightly after 2 weeks (Fig. 2C). Interestingly, 


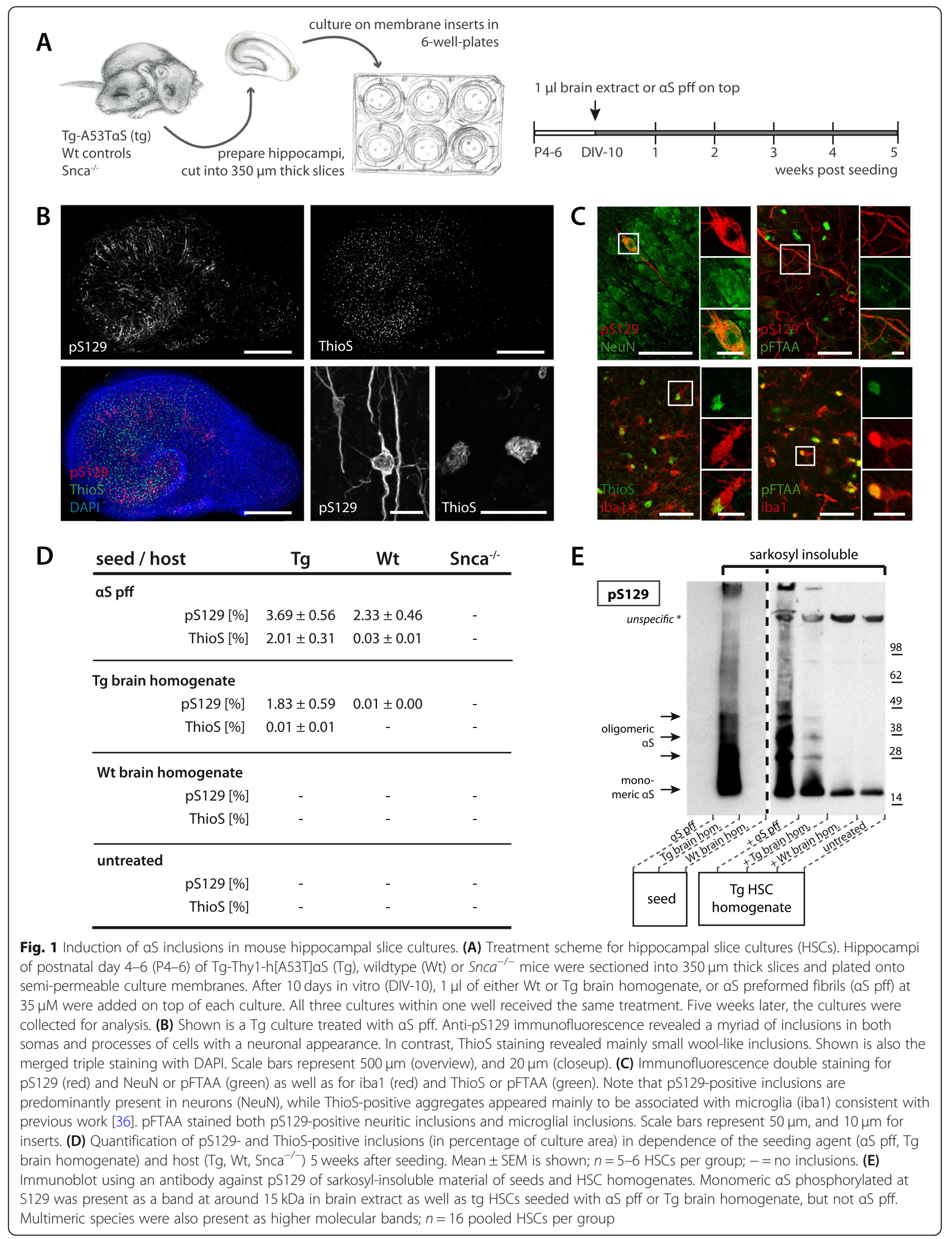


microglial inclusion appearance was delayed and occurred first after 2 weeks and increased thereafter (Fig. 2D, E). To investigate the temporal appearance of $\alpha \mathrm{S}$ lesions in vivo, $\mathrm{tg}$ brain extract seeding in adult mice of the same genotype (Thy1-h[A53T] $\alpha \mathrm{S}$ tg mice) was performed. Here, we observed an increasing quantity of lesions which are not reaching to plateau within 30 days attributed to the fewer seeds in tg brain extract (Supplementary Fig. 4).

\section{aS lesion-related neurodegeneration can be monitored by $\mathrm{NfL}$ release in culture medium}

A sensitive measure for neurodegeneration is the thinning of the cultures. A clear degenerative phenotype was observed in tg HSCs with the highest concentration of $\alpha \mathrm{S}$ pff treatment $(350 \mu \mathrm{M})$ (Supplementary Fig. 5). To assess the extent of subtler neurodegeneration, the release of neurofilament light (NfL) into the culture medium was measured. Increased levels of NfL have previously been found in CSF and blood of Thy1$\mathrm{h}[\mathrm{A} 53 \mathrm{~T}] \alpha \mathrm{S}$ tg mice and has been linked to the induction and spreading of $\alpha \mathrm{S}$ lesions [38]. Indeed, an increase in NfL upon induction of $\alpha S$ pathology was also found in tg HSCs treated with $35 \mu \mathrm{M} \alpha \mathrm{S}$ pff (Fig. 3A, B).

A link between the induced $\alpha \mathrm{S}$ pathology and the increase in NfL release was demonstrated by blocking $\alpha \mathrm{S}$ lesion formation. Three weeks -after mixing and treating cultures with human anti- $\alpha \mathrm{S}$ antibody and $\alpha \mathrm{S}$ pff, analysis revealed greatly reduced neuronal and microglial inclusions (Fig. 3C - E) and a concomitant attenuation of the $\mathrm{NfL}$ concentration in the culture medium (Fig. $3 \mathrm{~F}$ ).

\section{Seed-induced synucleinopathy propagates to interconnected regions}

To establish HSCs as a model system to study the propagation of $\alpha \mathrm{S}$ aggregates, ATTO-550-labelled $\alpha \mathrm{S}$ pff were injected into the CA3 region (Fig. 4A) in order to observe whether locally induced $\alpha \mathrm{S}$ inclusions spread to the distant CA1 via Schaffer collaterals and/or retrogradely via mossy fibres to the dentate gyrus (DG). Examination revealed the formation of pS129-positive inclusions in neurites around the CA3 injection site already at day 4 with some early spreading to the DG (Fig. 4B). At 7 days post-injection, both DG and CA1 neurons exhibited somatic $\alpha S$ inclusions with neuritic inclusions along the Schaeffer collaterals and mossy fibres (Fig. 4C), suggesting both anterograde and retrograde spreading [40].

To rule out the possibility of diffusion of the applied seeds from the injection area in CA3 to DG and CA1, Schaeffer collaterals and mossy fibres were surgically disconnected. As a result, virtually no spreading across the incision site was apparent (Fig. 4D). Presuming that a simple mechanic cut would not impair diffusion of seeds, these results indicate that the observed formation of $\alpha \mathrm{S}$ inclusions in distal regions spreading occurs via neural connection.

\section{Spreading of aS inclusions can be blocked by anti-aS antibody}

Our initial observation that the $\alpha \mathrm{S}$ antibody blocks seeding once mixed with $\alpha \mathrm{S}$ pff (see Fig. 3) urged us to study whether the antibody was also able to block seeding and propagation of $\alpha \mathrm{S}$ lesions when present in the culture medium (Fig. 4E - O). When the medium was continuously supplemented with $\alpha \mathrm{S}$ antibody starting 7 days before $\alpha \mathrm{S}$ pff injections, there was a robust blocking of $\alpha \mathrm{S}$ lesions 1 week after seed application (Fig. 4E - G). However, when $\alpha S$ antibody was added to the medium $1 \mathrm{~h}$ after injection of $\alpha \mathrm{S}$ pff, no reduction of $\alpha \mathrm{S}$ lesions were observed at day 7 (Fig. $4 \mathrm{H}-\mathrm{J}$ ).

We then assessed the ability of the $\alpha \mathrm{S}$ antibody to prevent the spreading of pathology from the CA3 injection site throughout the culture. $\alpha \mathrm{S}$ pff seeds were injected into CA3 and, again, $1 \mathrm{~h}$ later, the culture medium was continuously supplemented with $\alpha \mathrm{S}$ antibody (Fig. 4K). Five weeks later, there was a decrease of neuronal pathology (Fig. 4L, M) suggesting an inhibiting effect of the $\alpha \mathrm{S}$ antibody on the spreading, particularly to CA1. A decrease in microglial inclusions was also apparent, but did not reach statistical significance (Fig. 4N, O).

\section{Induction of aS inclusions in adult human brain slice cultures}

Having established the conditions needed to induce $\alpha \mathrm{S}$ lesions in murine HSCs, we next addressed the possibility of adapting these findings to cultures derived from human adult brain tissue [29]. Such human cultures are derived from resected adult brain tissue and are stable in vitro for up to 21 days when cultured in human CSF $[29,41]$ (Fig. 5A-C). $\alpha \mathrm{S}$ pff were applied on top of each culture in the same concentration as previously used for the mouse cultures $(1 \mu \mathrm{l}, 35 \mu \mathrm{M})$. In contrast to the murine cultures, however, $\alpha \mathrm{S}$ pff were already applied after 3 days in culture (compared to 10 days for the murine culture) because of the overall shorter culturing timespan for human cultures. To mimic the tg mouse cultures, hA53T- $\alpha \mathrm{S}$ was also expressed in the human cultures (at day 1) using an adeno-associated virus (AAVhA53T $-\alpha \mathrm{S})$.

Consistent with the murine cultures, 1 week after the seed application, pS129-positive inclusions were apparent in AAV-hA53T- $\alpha$ S-treated human cultures (Fig. 5D, E). In contrast, lesions were absent in cultures treated with either $\alpha \mathrm{S}$ pff or AAV-hA53T- $\alpha \mathrm{S}$ alone, or in untreated controls (Fig. 5F). The pS129-positive inclusions were neuronal and also positive for pFTAA. No pFTAApositive microglial inclusions were observed at this time point. Similar to observations in murine cultures, the 

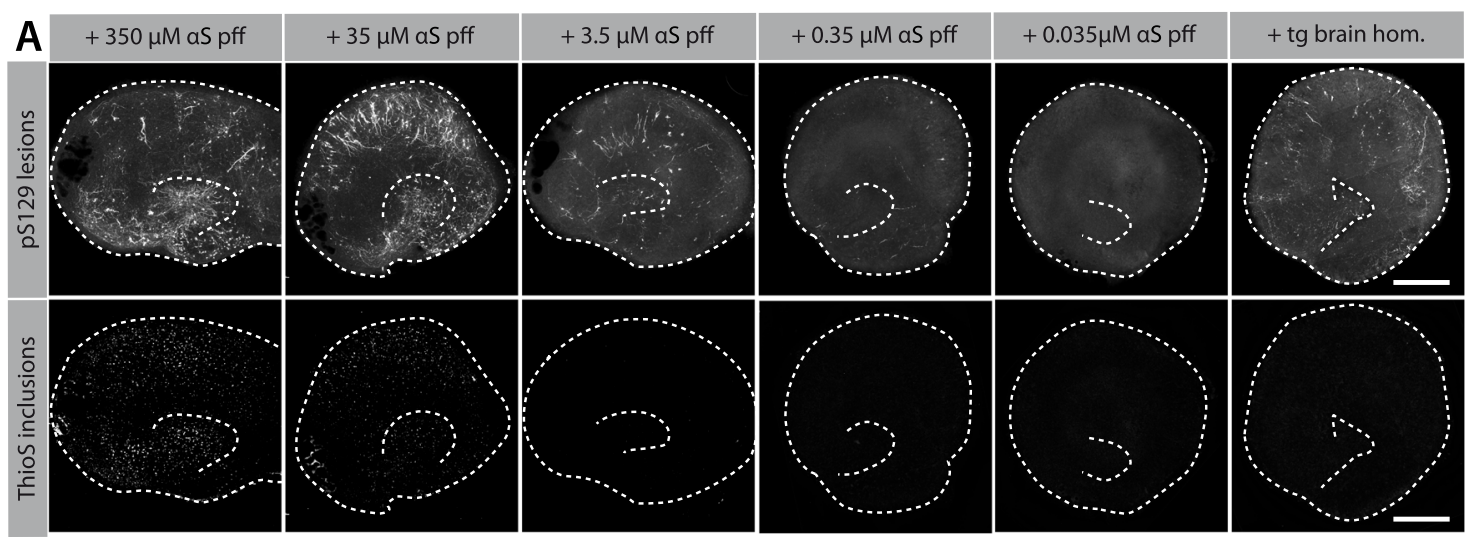

B


C

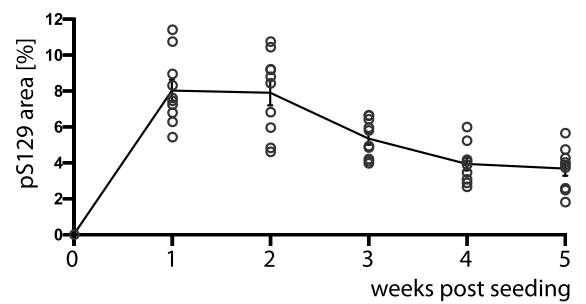

D

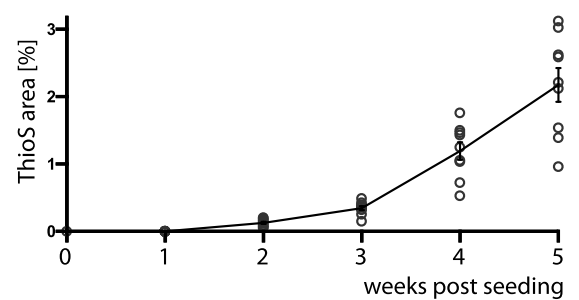

\section{$\mathrm{E}$}
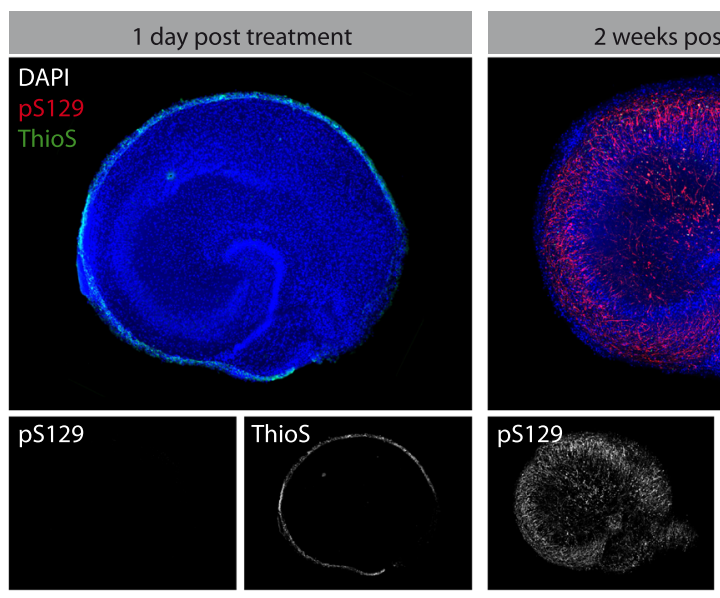

2 weeks post treatment

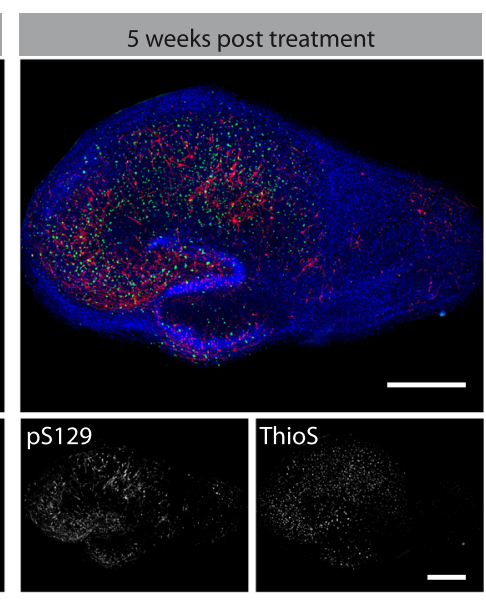

Fig. 2 Induction of aS inclusions is seed-, concentration- and time-dependent. See Fig. 1A for treatment scheme. (A) Immunofluorescence staining for pS129 and ThioS in tg HSCs at 5 weeks post-seeding. The concentration of aS pff (350 $\mu \mathrm{M}$ with subsequent 10- to 10,000-fold dilutions) determines the abundance of pS129-positive aS inclusions (largely neuronal) and ThioS-positive inclusions (largely microglial) in tg HSCs. Brain homogenate shows approximately the seeding activity of 1:100 aS pff. However, the seed concentration in the brain homogenate is likely much lower compared to the one of aS pff (see Results). Scale bars $=50 \mu \mathrm{m}$. (B) Normalised quantification of pS129-positive and ThioSpositive inclusions in $\mathrm{tg} \mathrm{HSCS}$ treated with different concentrations of aS pff (light grey bars) or with tg brain homogenate (dark grey bar). Mean \pm SEM is shown; $n=5-6$ HSCs per group. (C, D) Quantification of percentage of pS129-positive (C) and ThioS-positive (D) aS inclusions over time using $35 \mu \mathrm{M}$ aS pff. Mean \pm SEM are shown; $n=9$ HSCs per group and timepoint. (E) Distribution of pS129- and ThioS-positive aggregates in tg HSCs treated analysed at 1 day, 2 weeks or 5 weeks post-treatment. After 1 day, ThioS-positive aS pff (green) still surrounded the culture with no induction of aS lesions. At 2 weeks, pS129-positive neuronal inclusions (red) were abundant, but appeared to decline at 5 weeks post-seeding. In contrast, Thios-positive microglia inclusions increased from 2 to 5 weeks post-seeding. Scale bars represent $500 \mu \mathrm{m}$ 
induced $\alpha \mathrm{S}$ inclusions in these human cultures appeared as smooth, fibrillar structures in the cytoplasm with pathology also extending into the processes (Fig. 5D, E).

\section{Discussion}

Neurodegenerative diseases are characterised by proteopathic lesions which develop many years, if not decades before the first symptoms and lesions at death are likely to be different from the ones driving the disease [16]. Therefore, understanding the critical early phase of seeding and finding biomarkers to monitor disease progression require disease relevant models. Here we present $\alpha \mathrm{S}$ lesion development in a mouse hippocampal slice culture model and find that microglial inclusions and $\mathrm{NfL}$ release follow neuronal $\alpha \mathrm{S}$ inclusions. Moreover, we show that NfL release and spreading of $\alpha \mathrm{S}$ lesions can be inhibited by a human $\alpha \mathrm{S}$ antibody. Finally, we demonstrate that $\alpha \mathrm{S}$ lesions can also be induced in adult human brain slice cultures.

Previous efforts to develop organotypic murine slice cultures of synucleinopathies mainly used viral overexpression of $\alpha \mathrm{S}$ [42]. In this study, we expand these endeavours and show that one-time seeding of brain-derived or synthetic $\alpha \mathrm{S}$ seeds is sufficient for the induction and subsequent spreading of $\alpha \mathrm{S}$ lesions in murine hippocampal slice cultures. Neuronal $\alpha S$ inclusions in the cultures appear mature and their biochemical properties (phosphorylation of serine 129 and sarkosyl insolubility) similar to previously observed in cultures [43, 44], as wells as $\alpha S$ transgenic or wildtype mice in which the lesions have been induced by seeded aggregation [9-12]. The present findings of slower $\alpha \mathrm{S}$ lesions induction and overall lower burden of pathology in wt compared to tg cultures are in line with observations in mouse models $[9,10]$ and can be linked to enhanced vulnerability of neuronal populations with high $\alpha \mathrm{S}$ expression levels $[45,46]$. The morphology of the induced neuronal $\alpha \mathrm{S}$ lesions in the cultures was dependent on the $\alpha S$ seed type ( $\alpha S$-tg mouse brainderived or synthetic $\alpha \mathrm{S}$ pff), again, similar to what has been described for seeded induction in mice [9-11, 4749]. These observations are consistent with prion-like templated propagation [4] and structural differences between pff and brain-derived human $\alpha \mathrm{S}$ seeds [50]. In the slice cultures as well as in mice, highly concentrated $\alpha S$ pff are more seeding active compared to the tg mouse brain material. Hence, when comparing the specific activity of $\alpha \mathrm{S}$ pff with tg brain-derived aggregates (seed activity per $\alpha S$ molecules), brain-derived $\alpha \mathrm{S}$ seeds appear to be more potent in seeding aggregation consistent with other proteopathic seeds [16].

The finding that $\alpha \mathrm{S}$ lesions in murine slice cultures are positive for the amyloid-binding dye pFTAA is in line with recent data in humans using pFTAA and other luminescent-conjugated oligothiophenes [51, 52]. This not only confirms the amyloid nature of the inclusions in the cultures, but it also opens up the possibility to use these fluorescent dyes for live imaging of $\alpha \mathrm{S}$ lesions and for spectral discrimination of $\alpha \mathrm{S}$ conformers [52-54].

A growing body of research has pinpointed the importance of neuroinflammation as an essential contributor to the pathogenesis of synucleinopathies [55]. As recently reported for $\alpha S$ tg mice [36], we also found in the cultures pFTAA- and ThioS-positive (but largely pS129-negative) inclusions in microglia. However, microglial inclusions developed with a delay of 2-3 weeks compared to the neuronal inclusions. The local appearance of the microglia inclusions was always linked to the neuronal inclusions both in magnitude and location. Intriguingly, targeting $\alpha \mathrm{S}$ seeds with an $\alpha \mathrm{S}$ antibody blocked both, neuronal and microglial inclusions. Although final proof of the nature and conformation of the microglia inclusions remains to be established, the present observations are consistent with the view that the microglial inclusions contain $\alpha \mathrm{S}$ of neuronal origins [36] and that microglia are somehow involved in the spreading of $\alpha$ S lesions in brain [56].

Modelling proteopathic lesions in slices with a brainlike environment bears the advantage that the cell-tocell transfer of $\alpha \mathrm{S}$ aggregates between interconnected regions may occur in a similar way to that in vivo $[16,57]$. Immunotherapy targeting aggregated $\alpha \mathrm{S}$ is a vigorously pursued therapeutic strategy, although its mechanism of action remains to be further investigated. Since it is not expected that antibodies readily enter intact neurons, $\alpha S$ antibodies that neutralise $\alpha S$ seeds are thought to capture the seeds extracellularly when transferred from cell to cell [58-62]. The observation that an $\alpha \mathrm{S}$ antibody was capable of reducing the number of $\alpha \mathrm{S}$ inclusions in DG and CA1 where the $\alpha \mathrm{S}$ lesions spread, but less so in the CA3 injection area, suggests that this human antibody can inhibit the spreading of $\alpha S$ seeds. Although, the current results do not provide a conclusive explanation for the mechanisms involved, our results demonstrate the utility of this culture system for studying $\alpha \mathrm{S}$ targeting disease-modifying drugs.

Early biomarkers that indicate the initial development of proteopathic lesions before the occurrence of clinical symptoms are essential for early diagnosis and disease progression monitoring. An increase of NfL in CSF and blood has been observed in $\alpha \mathrm{S}$-tg mice as well as in human synucleinopathies $[38,63]$. The present findings that $\alpha \mathrm{S}$ lesions and associated neurodegeneration in murine slice cultures can be monitored by measuring NfL levels in the culture medium reveals the culture system as an important tool for translational research. NfL measurements in cultures provide even some advantages over animal models (i.e. NfL in CSF) since longitudinal measurements are possible and changes of NfL levels 


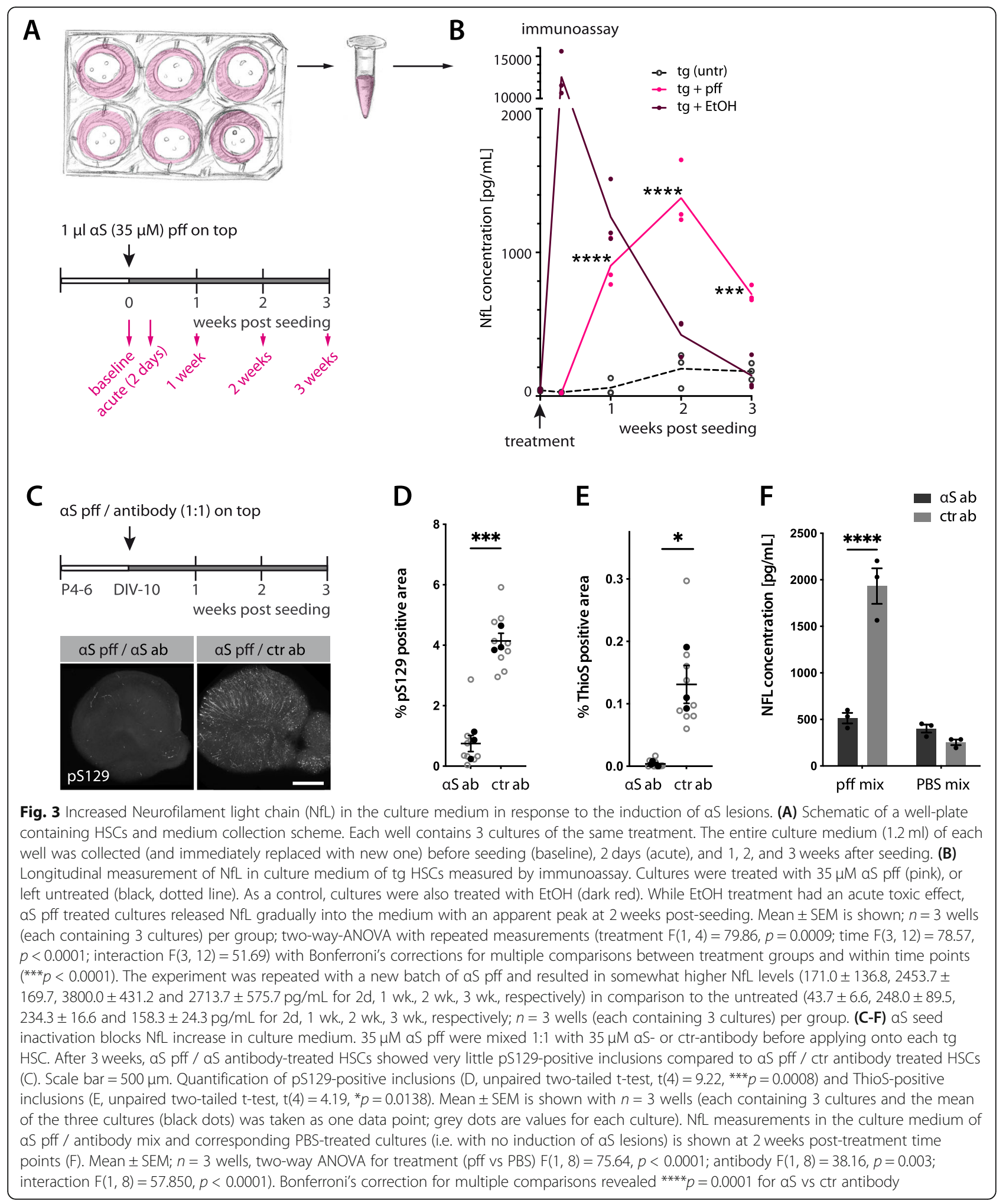


A

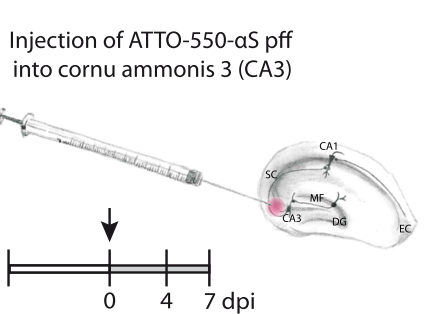

E

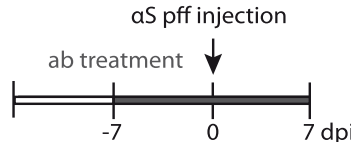

F

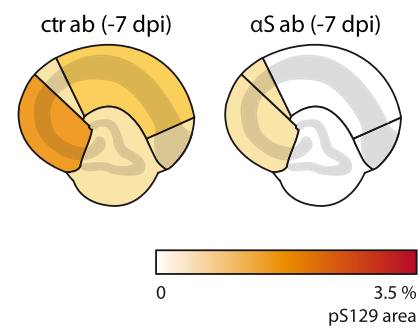

K



L

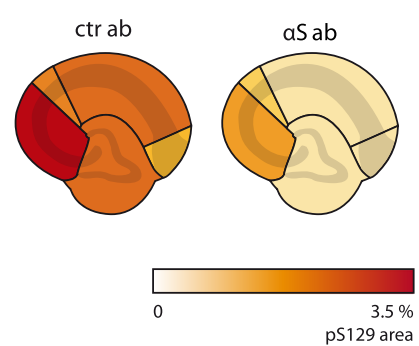

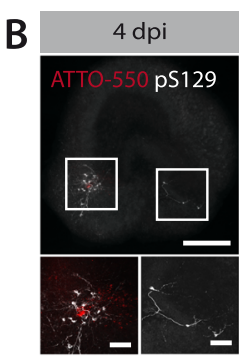
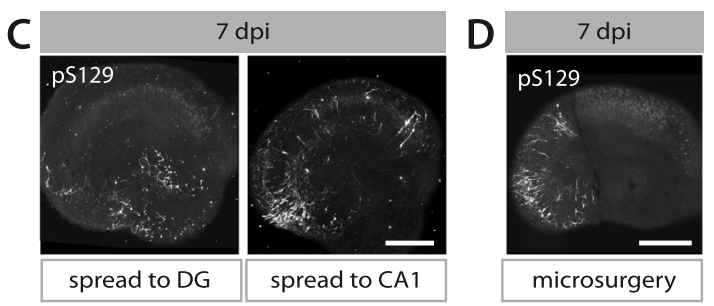
G ctrab (-7 dpi)
aS ab (-7 dpi)
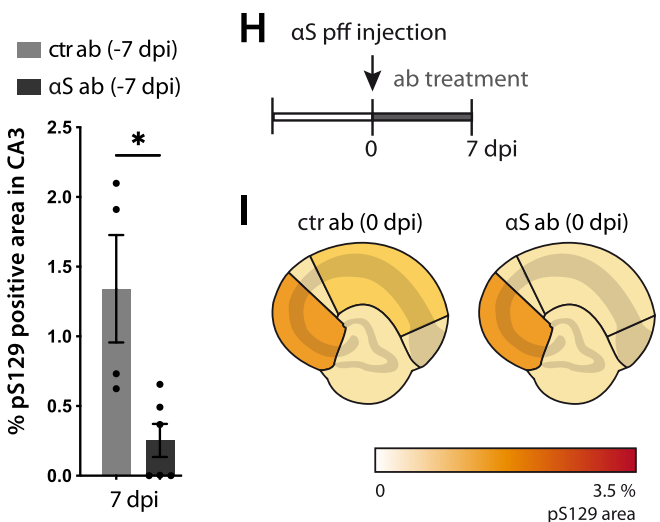

M

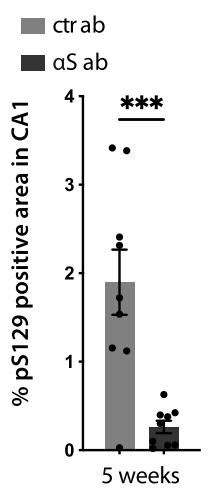

$\mathbf{N}$


J

$$
\begin{aligned}
& \text { ctrab (0 dpi) } \\
& \text { aS ab (0 dpi) }
\end{aligned}
$$

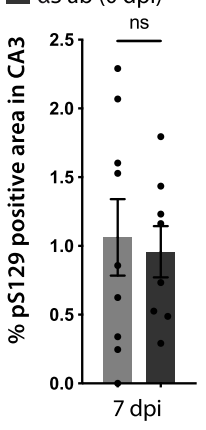

O

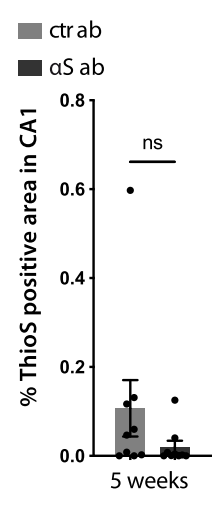

Fig. 4 Spreading of aS lesions in mouse hippocampal slice cultures. (A) Schematic illustration of the local injection paradigm of ATTO-550labelled aS pff (from the $35 \mu \mathrm{M}$ stock solution) into hippocampal CA3 region. (B) ATTO-550-aS pff (red) and the first neuritic pS129-positive inclusions (white) at the injection site (CA3) and in the dentate gyrus (DG) at 4 dpi. Note that as pff seeds are not phosphorylated upon uptake $[32,39]$ and therefore the pS129-positive signal can be attributed to aggregated endogenous aS. Scale bars $=500 \mu \mathrm{m}$ and $100 \mu \mathrm{m}$ (inserts). (C) Apart from the injection site (CA3) and DG, pS129-positive inclusions also appeared in CA1 at 7 dpi. Scale bars $=500 \mu m$. (D) Disconnecting CA3 from both CA1 and DG via microsurgery before injecting pff inhibited the spreading of the pathology at 7 dpi. Scale bars $=500 \mu \mathrm{m}$. (E-G) Antibody-mediated blocking of aS lesion induction. Schematic illustration of experimental setup (E). Seven days prior to aS pff injection into CA3, anti-aS or ctr antibodies were added to the medium (reaching a final concentration of $350 \mathrm{nM}$ ) and antibody treatment was continued until 7 dpi. Heatmap of pS129-positive area revealed largely reduced pS129-positive inclusions (F). Quantification of pS129-positive inclusions in CA3 (G). Mean \pm SEM; $n=6$ and $4 \mathrm{HSCs} /$ group for the aS and ctr antibody, respectively (2 HSCs from ctr group were excluded due to accidental injection into CA1 region); unpaired two-tailed t-test $\left(\mathrm{t}(\mathrm{S})=3.211, p=0.0124,{ }^{*} p<0.05\right)$. (H-J) Delayed antibody-treatment fails to block aS lesion induction. Schematic illustration of experimental setup $(H)$. Antibodies were added to the culture medium $1 \mathrm{~h}$ after aS pff injection into CA3 and antibody treatment was continued until $7 \mathrm{dpi}$. Heatmap revealed that such delayed antibody treatment did not prevent pS129-positive inclusions in injection region (I). Quantification of pS129-positive inclusions in CA3. Mean \pm SEM; $n=9$ HSCs/group; unpaired two-tailed t-test ( $\mathrm{t}(14)=0.7102$, $p=0.4892)$. (K-O) Antibody-mediated blocking of the spreading of the aS lesion. Schematic illustration of experimental setup (K). Antibodies were applied $1 \mathrm{~h}$ after aS pff injection into CA3 and antibodies were continuously added until 5 weeks post-seeding. Heatmap revealed that spreading of pS129-positive inclusions to other subregions has been largely reduced $(L)$ and the same was true for ThioS-positive inclusions (that start to develop 2-3 weeks after the neuronal inclusions, see Fig. 2) (N). Quantification of pS129-positive inclusions (M) and ThioS-positive inclusions (O) in CA1. Mean \pm SEM; $n=9$ HSCs/group; for pS129 (unpaired two-tailed t-test (t(16) $\left.=4.362,{ }^{* * *} p=0.0005\right)$; for ThioS, two-tailed Mann-Whitney test $(U=25, p=0.1782)$ 


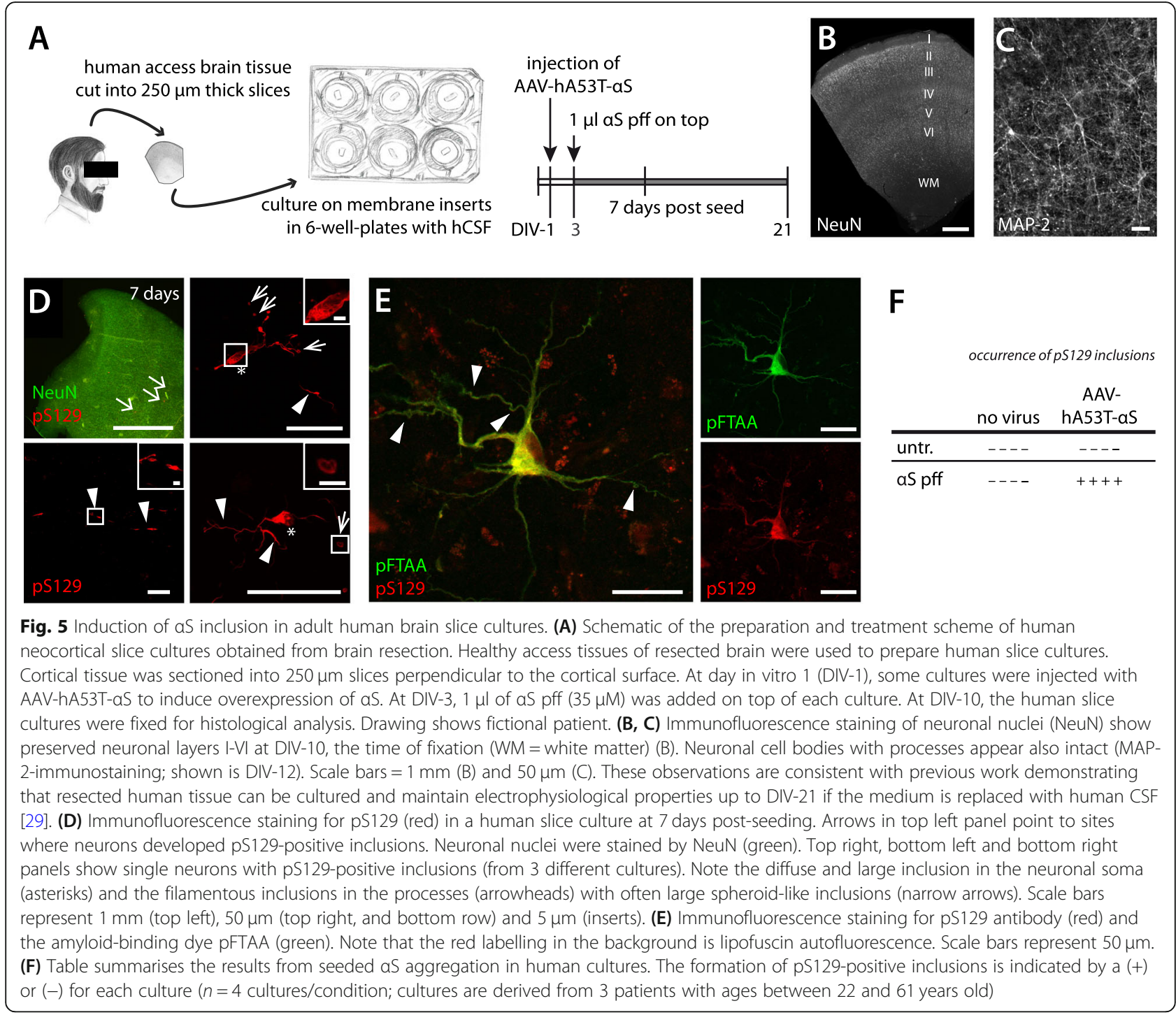

can be directly related to lesions as well as to therapeutic efforts.

While murine slice cultures now allow to study the formation, spreading, and targeting of the inclusions in a brain-like environment, it is important to acknowledge their limitations given that these cultures are derived from postnatal brain and therefore lack the aspect of aging. Furthermore, recent transcriptome studies revealed differences in disease-relevant genes between mouse and human cell populations [64-68]. Thus, the here described proof-of-principle translation of the slice culture model from mouse to human appears a first step forward and will allow to confirm findings derived from mouse cultures in a true aged human brain environment.

Human long-term slice cultures have only recently been developed [29,69]. The cultures used in the present study were derived from three individuals (22-61 years old) of both sexes. Since this small sample size does not allow any conclusions about the effects of age and gender, such parameters could be addressed in the future by increasing the donor sample size. In particular, it will be interesting to study whether $\alpha \mathrm{S}$ lesion induction is more prominent in aged donors compared to younger donors. Other open questions are whether longer culturing of the human cultures will allow the induction of $\alpha \mathrm{S}$ lesions without additional overexpression of A53T- $\alpha \mathrm{S}$ and whether microglia inclusions will also develop at prolonged culturing.

The induction of $\alpha \mathrm{S}$ lesions in adult human brain cultures is an exciting step forward. However, limitations remain and need to be overcome before the human culture model can be used in a routine lab environment. The availability of suited resection tissue is limited and long-term cultivation of human cultures appears more difficult compared to the murine cultures. In addition, human CSF is needed to nurture the human cultures 
whose availability is also limited. Thus, at present, the human slice culture model is best used to confirm findings from the mouse cultures in a true aged human brain environment.

\section{Conclusion}

We have established a quantitative cellular model system for studying $\alpha$-synucleinopathy that contains essential elements of in vivo tissue complexity and replicates key aspects of disease. We further provided proof-of-principle evidence for their clinical application in screening antibodies that prevent the spread of $\alpha \mathrm{S}$ lesions. As was the case in animal models and in humans, NfL measurements can be used as reliable readouts of disease in these slice cultures. Given the limitations of studying human disease in the context of an animal brain, we successfully translated this model from mouse to human and report the first induction of human $\alpha \mathrm{S}$ lesions in a true adult human brain environment. Although our study was focussed on the induction of $\alpha$-synucleinopathies, these slice culture models can be expanded to other proteopathies. Slice cultures allow for easy access and manipulation of the tissue while keeping the 3Rs (reduce, refine, replace) guiding principles. In particular, live-imaging of the pathology development with fluorescent amyloid dyes combined with single cell transcriptomics offer great potential to study human neurodegenerative diseases in new light.

\section{Abbreviations}

AB: Amyloid-beta; aS: Alpha-synuclein; AAV: Adeno-associated virus; CA: Cornu ammonis; CSF: Cerebrospinal fluid; DG: Dentate gyrus; DLB: Dementia with Lewy bodies; HSC: Hippocampal slice culture; MSA: Multiple system atrophy; NfL: Neurofilament light chain; PD: Parkinson's disease; PFA: Paraformaldehyde; PFF: Preformed fibrils; TG: Transgenic; WB: Western blot; WT: Wild type

\section{Supplementary Information}

The online version contains supplementary material available at https://doi. org/10.1186/s13024-021-00471-2

\begin{abstract}
Additional file 1: Supplementary Fig. 1. aS pff characterization. (A, B) Proteolytic profiles of monomeric (A) and fibrillar (B) aS (100 M in PBS) after proteinase $\mathrm{K}$ treatment $(3.7 \mu \mathrm{g} / \mu \mathrm{l})$ at $37^{\circ} \mathrm{C}$. Aliquots were removed from the reaction at the time indicated (in $\mathrm{min}$ ), immediately denatured with Laemmli buffer at $90^{\circ} \mathrm{C}$ for $5 \mathrm{~min}$ and analysed on 15\% PAGE. The gels were stained with Coomassie blue. The molecular weight markers are shown on the left of each gel. The as fibrils reveal the typical proteolytic pattern as reported previously [30, 31]. (C, D) Transmission electron micrographs of the aS fibrils before (C) and after fragmentation (D). The scale bar represents $200 \mathrm{~nm}$.
\end{abstract}

Additional file 2: Supplementary Fig. 2 Induction of aS aggregation is dependent on endogenous aS expression. Immunofluorescence staining for pS129 showed inclusions in Thy1-h[A537]aS tg HSCs seeded with Thy1-h[A53T]aS tg brain homogenate. In contrast, there was no induction of aS aggregation in Snca-/- HSCs after seeding using the same brain homogenate as seed. Analysis was done 5 weeks after treatment. See Fig. 1 for methodological detail. The experiment was done twice with 3 cultures each. Scale bar $=500 \mu \mathrm{m}$.

Additional file 3: Supplementary Fig. $\mathbf{3}$ aS aggregates in aS pff and tg brain homogenate. (A, B) HTRF-FRET immunoassay analysis of aggregated aS in aS pff (light grey), tg brain homogenate (dark grey), and wt homogenate (white) at different sample dilutions. Data is reported as ratio of $665 \mathrm{~nm} / 620 \mathrm{~nm} \times 10,000$. Note that aS pff showed Hook effect at dilutions < 1: 51200 (Hook effect: high aggregate concentrations will capture all antibodies leading to a plateau and to a decrease of the signal) (A). Dotted line (red) illustrates exemplary comparison of dilutions that resulted in similar aggregation signal (B). Aggregation levels that gave a signal ratio of $\sim 20,000$ for ratio of $665 \mathrm{~nm} / 620 \mathrm{~nm} \times 10,000$. aS pff (light grey) needed to be diluted approximately 204,800 -fold, and tg brain homogenate (dark grey) 25-fold. Although the signal cannot be attributed to an absolute amount of aggregated as given that the conformations of aggregated aS in pff and tg brain extract are likely to be different, results appear consistent with tg brain-derived as seeds to be more seeding potent compared to aS pff seeds. Mean $\pm \mathrm{SEM} ; n=3$ triplicate measurements per dilution.

Additional file 4: Supplementary Fig. 4. Time course of seeded aS aggregation in Thy $1-h[$ A53T]aS tg mice. (A, B) Immunohistochemistry for pS129 (black) of DG from Thy1-h[A53T]aS tg mice injected at 3-4 months of age with Thy1-h[A53T]aS tg brain homogenate (A) or wt brain homogenate (B) at one day post-injection (dpi), 7 dpi or $30 \mathrm{dpi}$. For each group, three mice were used. Sections were counter stained using nuclear fast red. Note that the same brain homogenates were used for slice culture experiments. (A) First inclusions appeared around $7 \mathrm{dpi}$ in all three mice and became more abundant by 30 dpi. (B) In contrast, no inclusions were found in wt brain homogenate-treated mice at any time point analysed. Scale bar represents $100 \mu \mathrm{m}$.

Additional file 5: Supplementary Fig. 5. Treatment with highly concentrated aS pff is neurotoxic to Thy1-h[A53T]aS tg HSCs. (A) Immunostaining of pS129-positive aggregates in $50 \mu \mathrm{m}$-horizontal sections from Thy1-h[A53T]aS tg HSCs treated with $350 \mu \mathrm{M}$ aS pff at 1, 2 and 5 weeks post-seeding. Scale bar $=500 \mu \mathrm{m}$. (B) Measurement of culture thickness (in $\mu \mathrm{m}$ at the time of fixation) in untreated, $350 \mu \mathrm{M}$ aS pfftreated and $35 \mu \mathrm{M}$ aS pff-treated tg HSCs. Mean \pm SEM; n(untr., 1 week) = 6, n(untr., 3 weeks $)=18, \mathrm{n}$ (untr., 5 weeks $)=19, \mathrm{n}(35 \mu \mathrm{M}$ pff, 1 week $)=15$, $\mathrm{n}(35 \mu \mathrm{M}$ pff, 2 weeks $)=10, \mathrm{n}(35 \mu \mathrm{M}$ pff, 3 weeks $)=31, \mathrm{n}(35 \mu \mathrm{M}$ pff, 4 weeks $)=9, \mathrm{n}(35 \mu \mathrm{M}$ pff, 5 weeks $)=18, \mathrm{n}(350 \mu \mathrm{M}$ pff, 1-4 weeks $)=6$ each, $\mathrm{n}(350 \mu \mathrm{M}$ pff, 5 weeks $)=35 \mathrm{HSCs}$ per group. Note that culture thickness was routinely measured and results come from many different experiments, which accounts for the differences among the n/group. (C) Violin graphs displaying thickness of tg HSCs across different treatments at 5 weeks post-treatment. $\mathrm{n}(\mathrm{tg}, \mathrm{untr})=19,. \mathrm{n}(\mathrm{tg}, \mathrm{tg}$ brain hom. $)=24, \mathrm{n}(\mathrm{tg}$, $35 \mu \mathrm{M}$ pff $)=18, \mathrm{n}(\mathrm{tg}, 350 \mu \mathrm{M} \mathrm{pff})=35$. Kruskal-Wallis test $(\mathrm{H}=45.82 ; \mathrm{p}<$ $0.0001):{ }^{*} p<0.0001$ Dunn's multiple comparisons against untreated HSCs. (D) Violin graphs displaying thickness of wt HSCs across different treatments at 5 weeks post-treatment. $n(w t$, untr. $)=21, n(w t$, tg brain hom. $)=27, \mathrm{n}(\mathrm{wt}, 35 \mu \mathrm{M}$ pff $)=3, \mathrm{n}(\mathrm{wt}, 350 \mu \mathrm{M}$ pff $)=18$. Kruskal-Wallis test $(H=11.64 ; p=0.0087):{ }^{* *} p<0.01$ Dunn's multiple comparisons against untreated HSCS.

\section{Acknowledgements}

We would like to Peter R. Nilsson (Linköping, Sweden) for the generous donation of the amyloid-binding dye pFTAA, Frank Heppner and Shirin Schneeberger (Charité) for helping with the Thy1-h[A53T]aS mouse colony, Marina Karakhanyan (Hertie Institute, Tuebingen) for Snca-- mouse breeding, Bernd Heimrich (Freiburg, Germany), Lary Walker (Atlanta, GA, USA), Manuela Neumann (DZNE, Tuebingen) and all the members of our departments for experimental help and comments to this manuscript. D.K.-V. is in receipt of an Alexander von Humboldt-Foundation research fellowship.

\section{Authors' contributions}

M.Barth, R.N., J.B., M.W., S.M, and G.T. prepared hippocampal slice cultures and M. Barth, M. Bacioglu, R.N., and G.T. designed and performed treatment experiments; M.Barth, M. Bacioglu designed and performed the biochemical work; N.S., T.V.W. and H.K prepared human slice cultures, and G.T. designed and performed treatment experiments; M. Bacioglu and M.S. designed and performed in vivo seeding experiments; L.M.H. performed immunoassays for alpha-synuclein and neurofilament light; K.F. and M.A. contributed antibodies and provided experimental input and performed CISBIO assay; A.A. and R.M. contributed synuclein pre-formed fibrils; D.R.S. provided A53T mouse model; P.J.K. provided Snca ${ }^{-1-}$ mouse; M. Barth, M. Bacioglu, R.N., M.J., and G.T. 
designed the overall study, and together with D.K.-V. wrote the manuscript All other co-authors edited the manuscript. All authors read and approved the final manuscript.

\section{Funding}

This work has received support from the EU/EFPIA/Innovative Medicines Initiative 2 Joint Undertaking (IMPRiND grant No 116060). This project has been made possible in part by a grant to GT from the funds provided by the Alzheimer Forschung Initiative e.V. (20019p) and to H.K. and D.K.-V. from the Chan Zuckerberg Initiative DAF (2020-221779). Open Access funding enabled and organized by Projekt DEAL.

\section{Availability of data and materials}

The datasets are available from the corresponding author upon reasonable request.

\section{Declarations}

\section{Ethics approval and consent to participate}

Animal and human materials and experimental procedures were approved by the local Animal Care and Use Committees and the ethics committee of the University of Tuebingen as well as written informed consent was obtained from all patients.

\section{Consent for publication}

All involved parties consented to publication of this work.

\section{Competing interests}

K.F, M.A. are current employees of Lundbeck. D.S. is a current employee of Novartis. All authors declare no competing interest.

\section{Author details}

DZNE, German Center for Neurodegenerative Diseases, 72076 Tuebingen, Germany. ${ }^{2}$ Department of Cellular Neurology, Hertie Institute for Clinical Brain Research, University of Tuebingen, 72076 Tuebingen, Germany. ${ }^{3}$ Graduate Training Center of Neuroscience, University of Tuebingen, 72076 Tuebingen, Germany. ${ }^{4}$ Department of Neurology and Epileptology, Hertie Institute for Clinical Brain Research, University of Tuebingen, 72076 Tuebingen, Germany. 'Department of Neurosurgery, University of Tuebingen, 72076 Tuebingen, Germany. ${ }^{6}$ Division of Neuroscience, H. Lundbeck A/S, 2500 Valby, Denmark. ${ }^{7}$ MIRCen, CEA and Laboratory of Neurodegenerative Diseases, CNRS, Institut François Jacob, 92265 Fontenay-aux-Roses, France. ${ }^{8}$ Laboratory of Functional Neurogenetics, Department of Neurodegeneration, Hertie-Institute for Clinical Brain Research, University of Tuebingen, 72076 Tuebingen, Germany. ${ }^{9}$ Neuroscience Research, Novartis Institutes for BioMedical Research, $\mathrm{CH}-4056$ Basel, Switzerland. ${ }^{10}$ Department of Epileptology, Neurology, RWTH Aachen University, Aachen, Germany.

Received: 9 April 2021 Accepted: 6 July 2021

Published online: 11 August 2021

\section{References}

1. Kordower JH, Chu Y, Hauser RA, Freeman TB, Olanow CW. Lewy body-like pathology in long-term embryonic nigral transplants in Parkinson's disease Nat Med. 2008;14(5):504-6. https://doi.org/10.1038/nm1747.

2. Li J, Englund $\mathrm{E}$, Holton $\mathrm{J}$, et al. Lewy bodies in grafted neurons in subjects with Parkinson's disease suggest host-to-graft disease propagation. Nat Med. 2008;14(5):501-3. https://doi.org/10.1038/nm1746.

3. Olanow W, Prusiner SB. Is Parkinson 's disease a prion disorder ? vol. 106: 2009. p. 12571-2.

4. Jucker M, Walker LC. Self-propagation of pathogenic protein aggregates in neurodegenerative diseases. Nature. 2013;501(7465):45-51. https://doi.org/1 0.1038 /nature 12481

5. Danzer KM, Krebs SK, Wolff M, Birk G, Hengerer B. Seeding induced by asynuclein oligomers provides evidence for spreading of a-synuclein pathology. J Neurochem. 2009;111(1):192-203. https://doi.org/10.1111/j.14 71-4159.2009.06324.x.

6. Desplats P, Lee H-J, Bae E-J, Patrick C, Rockenstein E, Crews L, et al. Inclusion formation and neuronal cell death through neuron-to-neuron transmission of -synuclein. Proc Natl Acad Sci. 2009;106(31):13010-5. https://doi.org/10.1 073/pnas.0903691106.
7. Volpicelli-Daley LA, Luk KC, Patel TP, Tanik SA, Riddle DM, Stieber A, et al. Exogenous a-Synuclein fibrils induce Lewy body pathology leading to synaptic dysfunction and neuron death. Neuron. 2011;72(1):57-71. https:// doi.org/10.1016/j.neuron.2011.08.033.

8. Fernandes JTS, Chutna O, Chu V, Conde JP, Outeiro TF. A novel microfluidic cell co-culture platform for the study of the molecular mechanisms of Parkinson's disease and other Synucleinopathies. Front Neurosci. 2016;10:111. https://doi.org/10.3389/fnins.2016.00511.

9. Luk KC, Kehm VM, Zhang B, O'Brien P, Trojanowski JQ, Lee VMY. Intracerebral inoculation of pathological a-synuclein initiates a rapidly progressive neurodegenerative a-synucleinopathy in mice. J Exp Med. 2012; 209(5):975-86. https://doi.org/10.1084/jem.20112457.

10. Luk KC, Kehm V, Carroll J, Zhang B, O'Brien P, Trojanowski JQ, et al. Pathological a-Synuclein transmission initiates Parkinson-like neurodegeneration in nontransgenic mice. Science. 2012;338(6109):949-53. https://doi.org/10.1126/science.1227157.

11. Masuda-Suzukake M, Nonaka T, Hosokawa M, Oikawa T, Arai T, Akiyama H, et al. Prion-like spreading of pathological a-synuclein in brain. Brain. 2013; 136(4):1128-38. https://doi.org/10.1093/brain/awt037.

12. Sacino AN, Brooks M, Shaw G, et al. Brain injection of a-Synuclein induces multiple proteinopathies, gliosis, and a neuronal injury marker. J Neurosci. 2014;34:12368-78. https://doi.org/10.1523/JNEUROSCI.2102-14.2014.

13. Schweighauser $M$, Bacioglu M, Fritschi SK, Shimshek DR, Kahle PJ, Eisele YS, et al. Formaldehyde-fixed brain tissue from spontaneously ill a-synuclein transgenic mice induces fatal a-synucleinopathy in transgenic hosts. Acta Neuropathol. 2015;129(1):157-9. https://doi.org/10.1007/s00401-014-1360-5.

14. Kim S, Kwon S-H, Kam T-I, Panicker N, Karuppagounder SS, Lee S, et al. Transneuronal propagation of pathologic a-Synuclein from the gut to the brain models Parkinson's disease. Neuron. 2019;103(4):627-641.e7. https:// doi.org/10.1016/j.neuron.2019.05.035.

15. Challis C, Hori A, Sampson TR, Yoo BB, Challis RC, Hamilton AM, et al. Gut-seeded asynuclein fibrils promote gut dysfunction and brain pathology specifically in aged mice. Nat Neurosci. 2020;23(3):327-36. https://doi.org/10.1038/s41593-020-0589-7.

16. Jucker M, Walker LC. Propagation and spread of pathogenic protein assemblies in neurodegenerative diseases. Nat Neurosci. 2018;21(10):1341-9. https://doi.org/10.1038/s41593-018-0238-6.

17. Rey NL, Steiner JA, Maroof N, et al. Widespread transneuronal propagation of a-synucleinopathy triggered in olfactory bulb mimics prodromal Parkinson's disease. J Exp Med. 2016;213(9):1759-78. https://doi.org/10.1084/jem.20160368.

18. Peng C, Gathagan RJ, Covell DJ, Medellin C, Stieber A, Robinson JL, et al. Cellular milieu imparts distinct pathological a-synuclein strains in a-synucleinopathies. Nature. 2018;557(7706):558-63. https://doi.org/10.1038/s41586-018-0104-4.

19. Yamada K, Iwatsubo T. Extracellular a-synuclein levels are regulated by neuronal activity. Mol Neurodegener. 2018;13(1):9. https://doi.org/10.1186/ s13024-018-0241-0.

20. Koh YH, Tan LY, Ng S-Y. Patient-derived induced pluripotent stem cells and organoids for modeling alpha Synuclein propagation in Parkinson's disease. Front Cell Neurosci. 2018;12:1-12. https://doi.org/10.3389/fncel.2018.00413.

21. Park J, Wetzel I, Marriott I, Dréau D, D'Avanzo C, Kim DY, et al. A 3D human triculture system modeling neurodegeneration and neuroinflammation in Alzheimer's disease. Nat Neurosci. 2018;21(7):941-51. https://doi.org/10.103 8/s41593-018-0175-4.

22. Gonzalez C, Armijo E, Bravo-Alegria J, Becerra-Calixto A, Mays CE, Soto C. Modeling amyloid beta and tau pathology in human cerebral organoids. Mol Psychiatry. 2018;23(12):2363-74. https://doi.org/10.1038/s41380-018-0229-8.

23. Mancuso R, Van Den Daele J, Fattorelli N, et al. Stem-cell-derived human microglia transplanted in mouse brain to study human disease. Nat Neurosci. 2019;22(12):2111-6. https://doi.org/10.1038/s41593-019-0525-x.

24. Hasselmann J, Coburn MA, England W, Figueroa Velez DX, Kiani Shabestari $\mathrm{S}$, Tu CH, et al. Development of a chimeric model to study and manipulate human microglia in vivo. Neuron. 2019;103(6):1016-1033.e10. https://doi. org/10.1016/j.neuron.2019.07.002.

25. van der Putten $\mathrm{H}$, Wiederhold KH, Probst A, Barbieri S, Mistl C, Danner S, et al. Neuropathology in mice expressing human alpha-synuclein. J Neurosci. 2000; 20(16):6021-9. https://doi.org/10.1523/JNEUROSCl.20-16-06021.2000.

26. Abeliovich A, Schmitz Y, Fariñas I, Choi-Lundberg D, Ho WH, Castillo PE, et al. Mice lacking alpha-synuclein display functional deficits in the nigrostriatal dopamine system. Neuron. 2000;25(1):239-52. https://doi.org/1 0.1016/s0896-6273(00)80886-7.

27. Mayer D, Fischer $H$, Schneider U, Heimrich B, Schwemmle M. Borna disease virus replication in Organotypic hippocampal slice cultures from rats results 
in selective damage of dentate granule cells. J Virol. 2005;79(18):11716-23. https://doi.org/10.1128/JVI.79.18.11716-11723.2005.

28. Novotny R, Langer F, Mahler J, Skodras A, Vlachos A, Wegenast-Braun BM, et al. Conversion of synthetic $A \beta$ to in vivo active seeds and amyloid plaque formation in a hippocampal slice culture model. J Neurosci. 2016;36(18): 5084-93. https://doi.org/10.1523/JNEUROSCI.0258-16.2016.

29. Schwarz N, Uysal B, Welzer M, Bahr JC, Layer N, Löffler H, et al. Long-term adult human brain slice cultures as a model system to study human CNS circuitry and disease. Elife. 2019;8:1-26. https://doi.org/10.7554/eLife.48417.

30. Bousset L, Pieri L, Ruiz-Arlandis G, Gath J, Jensen PH, Habenstein B, et al. Structural and functional characterization of two alpha-synuclein strains. Nat Commun. 2013;4(1):2575. https://doi.org/10.1038/ncomms3575.

31. Landureau M, Redeker V, Bellande T, Eyquem S, Melki R. The differential solvent exposure of $\mathrm{N}$-terminal residues provides 'fingerprints' of alphasynuclein fibrillar polymorphs. J Biol Chem. 2021;100737:100737. https://doi. org/10.1016/j.jbc.2021.100737.

32. Gribaudo S, Tixador P, Bousset L, Fenyi A, Lino P, Melki R, et al. Propagation of a-Synuclein strains within human reconstructed neuronal network. Stem Cell Rep. 2019;12(2):230-44. https://doi.org/10.1016/j.stemcr.2018.12.007.

33. Klingstedt T, Åslund A, Simon RA, Johansson LBG, Mason JJ, Nyström S, et al. Synthesis of a library of oligothiophenes and their utilization as fluorescent ligands for spectral assignment of protein aggregates. Org Biomol Chem. 2011;9(24):8356-70. https://doi.org/10.1039/c1ob05637a.

34. Ries J, Udayar V, Soragni A, Hornemann S, Nilsson KPR, Riek R, et al. Superresolution imaging of amyloid fibrils with binding-activated probes. ACS Chem Neurosci. 2013;4(7):1057-61. https://doi.org/10.1021/cn400091m.

35. Preische O, Schultz SA, Apel A, et al. Serum neurofilament dynamics predicts neurodegeneration and clinical progression in presymptomatic Alzheimer's disease. Nat Med. 2019;25(2):277-83. https://doi.org/10.1038/s41 591-018-0304-3.

36. Tanriöver G, Bacioglu M, Schweighauser M, Mahler J, Wegenast-Braun BM, Skodras A, et al. Prominent microglial inclusions in transgenic mouse models of a-synucleinopathy that are distinct from neuronal lesions. Acta Neuropathol Commun. 2020;8(1):133. https://doi.org/10.1186/s40478-020-00993-8.

37. Fujiwara H, Hasegawa M, Dohmae N, Kawashima A, Masliah E, Goldberg MS, et al. a-Synuclein is phosphorylated in synucleinopathy lesions. Nat Cell Biol. 2002;4(2):160-4. https://doi.org/10.1038/ncb748.

38. Bacioglu M, Maia LF, Preische O, Schelle J, Apel A, Kaeser SA, et al. Neurofilament light chain in blood and CSF as marker of disease progression in mouse models and in neurodegenerative diseases. Neuron. 2016;91(1):56-66. https://doi.org/10.1016/j.neuron.2016.05.018.

39. Pieri L, Chafey P, Le Gall M, et al. Cellular response of human neuroblastoma cells to a-synuclein fibrils, the main constituent of Lewy bodies. Biochim Biophys Acta. 2016;1860(1):8-19. https://doi.org/10.1016/j.bbagen.2015.10.007.

40. Brahic M, Bousset L, Bieri G, Melki R, Gitler AD. Axonal transport and secretion of fibrillar forms of a-synuclein, Aß42 peptide and HTTExon 1. Acta Neuropathol. 2016;131(4):539-48. https://doi.org/10.1007/s00401-016-1538-0.

41. Wickham J, Corna A, Schwarz N, Uysal B, Layer N, Honegger JB, et al. Human cerebrospinal fluid induces neuronal excitability changes in resected human neocortical and hippocampal brain slices. Front Neurosci. 2020;14:114. https://doi.org/10.3389/fnins.2020.00283.

42. Croft CL, Cruz PE, Ryu DH, Ceballos-Diaz C, Strang KH, Woody BM, et al. rAAV-based brain slice culture models of Alzheimer's and Parkinson's disease inclusion pathologies. J Exp Med. 2019;216(3):539-55. https://doi. org/10.1084/jem.20182184.

43. Elfarrash S, Jensen NM, Ferreira N, Betzer C, Thevathasan JV, Diekmann R, et al. Organotypic slice culture model demonstrates inter-neuronal spreading of alpha-synuclein aggregates. Acta Neuropathol Commun. 2019; 7(1):213. https://doi.org/10.1186/540478-019-0865-5.

44. Roux A, Wang X, Becker $\mathrm{K}$, Ma J. Modeling a-Synucleinopathy in Organotypic brain slice culture with preformed a-Synuclein amyloid fibrils. J Parkinsons Dis. 2020;10(4):1397-410. https://doi.org/10.3233/JPD-202026.

45. Courte J, Bousset L, Von Boxberg $Y$, et al. The expression level of alpha-synuclein in different neuronal populations is the primary determinant of its prion-like seeding. Sci Rep. 2020;10(1):4895. https:/doi.org/10.1038/541598-020-61757-x.

46. Taguchi K, Watanabe Y, Tsujimura A, Tatebe H, Miyata S, Tokuda T, et al. Differential expression of alpha-synuclein in hippocampal neurons. PLoS One. 2014;9(2):e89327. https://doi.org/10.1371/journal.pone.0089327.

47. Bétemps D, Verchère J, Brot S, Morignat E, Bousset L, Gaillard D, et al. Alphasynuclein spreading in M83 mice brain revealed by detection of pathological a-synuclein by enhanced ELISA. Acta Neuropathol Commun. 2014;2(1):29. https://doi.org/10.1186/2051-5960-2-29.

48. Tarutani A, Arai T, Murayama S, Hisanaga SI, Hasegawa M. Potent prion-like behaviors of pathogenic a-synuclein and evaluation of inactivation methods. Acta Neuropathol Commun. 2018;6(1):29. https://doi.org/10.1186/s40478-018-0532-2.

49. Lau A, So RWL, Lau HHC, Sang JC, Ruiz-Riquelme A, Fleck SC, et al. aSynuclein strains target distinct brain regions and cell types. Nat Neurosci. 2020;23(1):21-31. https://doi.org/10.1038/s41593-019-0541-x.

50. Goedert M, Hasegawa M, Schweighauser M, et al. (2020) Structures of asynuclein filaments from multiple system atrophy. bioRxiv. 2020.02.05. 935619. https://doi.org/10.1101/2020.02.05.935619.

51. Kuan WL, Stott K, He X, Wood TC, Yang S, Kwok JCF, et al. Systemic asynuclein injection triggers selective neuronal pathology as seen in patients with Parkinson's disease. Mol Psychiatry. 2019;26(2):1-12. https://doi.org/10.1 038/s41380-019-0608-9.

52. Shahnawaz M, Mukherjee A, Pritzkow S, Mendez N, Rabadia P, Liu X, et al. Discriminating a-synuclein strains in Parkinson's disease and multiple system atrophy. Nature. 2020;578(7794):273-7. https://doi.org/10.1038/s41 586-020-1984-7.

53. Klingstedt T, Ghetti B, Holton JL, Ling H, Nilsson KPR, Goedert M. Luminescent conjugated oligothiophenes distinguish between a-synuclein assemblies of Parkinson's disease and multiple system atrophy.Acta Neuropathol Commun. 2019;7(1):193. https://doi.org/10.1186/s40478-0190840-1.

54. Morgan SA, Lavenir I, Fan J, Masuda-Suzukake M, Passarella D, DeTure MA, et al. a-Synuclein filaments from transgenic mouse and human synucleinopathy-containing brains are major seed-competent species. J Biol Chem. 2020;295:jbc.RA119.012179(19):6652-64. https://doi.org/10.1074/jbc. ra119.012179.

55. Poewe W, Seppi K, Tanner CM, Halliday GM, Brundin P, Volkmann J, et al. Parkinson disease. Nat Rev Dis Prim. 2017;3(1):17013. https://doi.org/10.1038/ nrdp.2017.13

56. George S, Rey NL, Tyson T, Esquibel C, Meyerdirk L, Schulz E, et al. Microglia affect a-synuclein cell-to-cell transfer in a mouse model of Parkinson's disease. Mol Neurodegener. 2019;14(1):34. https://doi.org/10.1186/s13024-01 9-0335-3.

57. Peng C, Trojanowski JQ, Lee VM-Y. Protein transmission in neurodegenerative disease. Nat Rev Neurol. 2020;16(4):199-212. https://doi. org/10.1038/s41582-020-0333-7.

58. Masliah E, Rockenstein E, Adame A, Alford M, Crews L, Hashimoto M, et al. Effects of a-Synuclein immunization in a mouse model of Parkinson's disease. Neuron. 2005;46(6):857-68. https://doi.org/10.1016/j.neuron.2005.05. 010.

59. Tran HT, Chung CH-Y, Iba M, Zhang B, Trojanowski JQ, Luk KC, et al. aSynuclein immunotherapy blocks uptake and templated propagation of misfolded a-Synuclein and neurodegeneration. Cell Rep. 2014;7(6):2054-65. https://doi.org/10.1016/j.celrep.2014.05.033.

60. Masliah E, Rockenstein E, Mante M, Crews L, Spencer B, Adame A, et al. Passive immunization reduces behavioral and neuropathological deficits in an alpha-Synuclein transgenic model of Lewy bodydisease. PLoS One. 2011; 6(4):e19338. https://doi.org/10.1371/journal.pone.0019338.

61. Bae E-J, Lee H-J, Rockenstein E, Ho DH, Park EB, Yang NY, et al. Antibodyaided clearance of extracellular -Synuclein prevents cell-to-cell aggregate transmission. J Neurosci. 2012;32(39):13454-69. https://doi.org/10.1523/ JNEUROSCI.1292-12.2012.

62. Bergström A-L, Kallunki P, Fog K. Development of passive immunotherapies for Synucleinopathies. Mov Disord. 2016;31(2):203-13. https://doi.org/10.1 002/mds.26481.

63. Parnetti L, Gaetani L, Eusebi P, Paciotti S, Hansson O, el-Agnaf O, Mollenhauer B, Blennow K, Calabresi P (2019) CSF and blood biomarkers for Parkinson's disease. Lancet Neurol 18:573-586. https://doi.org/10.1016/S14 74-4422(19)30024-9, 6.

64. Zhou Y, Song WM, Andhey PS, Swain A, Levy T, Miller KR, et al. Human and mouse single-nucleus transcriptomics reveal TREM2-dependent and TREM2independent cellular responses in Alzheimer's disease. Nat Med. 2020;26(1): 131-42. https://doi.org/10.1038/s41591-019-0695-9.

65. Lin S, Lin Y, Nery JR, Urich MA, Breschi A, Davis CA, et al. Comparison of the transcriptional landscapes between human and mouse tissues. Proc Natl Acad Sci. 2014;111(48):17224-9. https://doi.org/10.1073/pnas.1413624111. 
66. Hodge RD, Bakken TE, Miller JA, Smith KA, Barkan ER, Graybuck LT, et al. Conserved cell types with divergent features in human versus mouse cortex Nature. 2019;573(7772):61-8. https://doi.org/10.1038/541586-019-1506-7.

67. Friedman BA, Srinivasan $\mathrm{K}$, Ayalon G, Meilandt WJ, Lin H, Huntley MA, et al. Diverse brain myeloid expression profiles reveal distinct microglial activation states and aspects of Alzheimer's disease not evident in mouse models. Cell Rep. 2018;22(3):832-47. https://doi.org/10.1016/j.celrep.2017.12.066.

68. Miller JA, Horvath S, Geschwind DH. Divergence of human and mouse brain transcriptome highlights Alzheimer disease pathways. Proc Natl Acad Sci. 2010;107(28):12698-703. https://doi.org/10.1073/pnas.0914257107.

69. Schwarz N, Hedrich UBS, Schwarz H, P.A. H, Dammeier N, Auffenberg E, et al. Human cerebrospinal fluid promotes long-term neuronal viability and network function in human neocortical organotypic brain slice cultures. Sci Rep. 2017;7(1):12249. https://doi.org/10.1038/s41598-017-12527-9.

\section{Publisher's Note}

Springer Nature remains neutral with regard to jurisdictional claims in published maps and institutional affiliations.

Ready to submit your research? Choose BMC and benefit from:

- fast, convenient online submission

- thorough peer review by experienced researchers in your field

- rapid publication on acceptance

- support for research data, including large and complex data types

- gold Open Access which fosters wider collaboration and increased citations

- maximum visibility for your research: over $100 \mathrm{M}$ website views per year

At $\mathrm{BMC}$, research is always in progress.

Learn more biomedcentral.com/submissions 